\title{
Peak Trekking of Hierarchy Mountain for the Detection of Cerebral Aneurysm using Modified Hough Circle Transform
}

\author{
Jubin Mitra*, Abhijit Chandra and Tanmay Halder \\ Department of Electronics \& Telecommunication Engineering \\ Bengal Engineering and Science University, Shibpur, Howrah, India
}

Received 12th Dec 2012; accepted 28th Jun 2013

\begin{abstract}
The Circle of Willis is in the junction of two carotid arteries and two vertebral arteries that supply the brain with nutrition. Junctions where these arteries come together may develop weak spots that can balloon out and fill with blood, creating aneurysms. These sac-like areas may leak or rupture, spilling blood into surrounding tissues which may cause artery spasm leading to potential stroke or even death. Clipping and coiling are two treatment options preferred by neurosurgeon which require proper detection of aneurysm. Medical practitioners are therefore emphasizing on the prior detection of cerebral aneurysm (CA) before rupture occurs leading to subarachnoid haemorrhage (SAH). This paper presents a novel method by application of Modified Hough Circle Transform \& Peak Trekking (MHCT-PT) technique on the image extracted from Digital subtraction angiography (DSA). Experimental results have firmly substantiated that the proposed method is highly efficient in properly detecting the location, size and type of aneurysm.
\end{abstract}

Key Words: 3D Parameter Space, Auto-Thresholding of CA images using Two Spin Operator, Cerebral Aneurysm (CA), Digital subtraction angiography (DSA), Medical Image Analysis, Midpoint Circle Algorithm, Modified Hough Circle Transform (MHCT), Modified Hough Circle Transform \& Peak Trekking (MHCT-PT) technique , Hough Hierarchy, Peak Trekking

\section{Introduction}

Cerebral aneurysm (CA) is a kind of neurological disorder where a portion of blood vessel in the brain weakens resulting in a bulging or ballooning out of part of the vessel wall. Aneurysms are most commonly found at the base of the brain just inside the skull [1], known as subarachnoid space and 90\% of subarachnoid haemorrhages (SAHs) are attributed mainly due to ruptured cerebral aneurysms [2]. This disorder generally results from congenital defects supported by other risky conditions like high blood pressure, atherosclerosis and less frequently by head trauma or infection. The out-come for patients treated before a ruptured aneurysm is much better than for those treated after. Therefore, the need for adequate evaluation of patients suspected of having a cerebral aneurysm is very important. Driven by this reason, proper detection of cerebral aneurysm entails as the primary task for the neurologists. Angiography, considered as a gold standard for evaluation of blood vessels of central nervous system, is widely used for the diagnosis of CA [3]. An angiogram imaging, besides providing

Correspondence to: $<$ jm61288@gmail.com $>^{*}$

Recommended for acceptance by $<$ Giorgio Fumera $>$

ELCVIA ISSN:1577-5097

Published by Computer Vision Center / Universitat Autònoma de Barcelona, Barcelona, Spain 
accurate visual information, also can help to detect the location and size of aneurysms and vascular malformations which forms the basis of this paper. Proper detection of CA has got a crucial clinical significance that might be useful in its prognosis and treatment trials. Intensity inhomogeneities and worst blood vessel contrast have been responsible for developing considerable difficulties in the automatic CA detection from angiography images.

Detection of CA from different modalities is a new domain, and has got much to be explored. One detection algorithm for cerebral aneurysms that works with 3D rotational angiography (3D RA), magnetic resonance angiography (MRA) and computed tomography angiography (CTA) data sets is first proposed by Hentschke $e t$ al. [4]. While the work of McKinney et al. [6] revealed that the combination of DSA with 3D RA is currently the most sensitive technique to detect untreated aneurysms and should be considered in suspicious cases of SAH where the aneurysm is not depicted by 64 multi-slice computed tomography angiography (64 MSCTA), because 64 MSCTA may occasionally miss aneurysms less than 3-4 mm size. While work of Lu et al. [7] claims that digital subtraction CT angiography has a high sensitivity and specificity in depicting intracranial aneurysms with different sizes and at different locations, compared with 3D DSA. But the work of Villablanca et al. [8], claims DSA is still a viable option for developing countries. So, our proposed method makes use of Auto-Thresholding along with Modified Hough Circle Transform \& Peak Trekking (MHCT-PT) technique for the accurate detection of CA from DSA. It also provides valuable information about the size of CA and its current state to rupture sooner or later.

The paper has been organized as follows: Section 2 presents a brief history of CA with different medical terminologies while Section 3 describes the DSA technique in brief. Section 4 illustrates how grayscale DICOM (Digital Imaging and Communications in Medicine) image is segmented to binary image, using our proposed Auto-Threshold algorithm. Section 5 describes in detail how our proposed novel technique MHCT-PT successfully segments and classifies aneurysm regions. The entire algorithm has been summarised in section 6. For proper functioning of the algorithm proper parameter optimizations are required as shown in section 7. Performance of the proposed algorithm with experimental results and related observations are analyzed in Sections 8 and 9 respectively, followed by some conclusive remarks in Section 10

\section{Cerebral Aneurysm}

Aneurysm is an abnormal swelling of a blood vessel due to weakness in the anatomy of the wall of the blood vessel. As blood pressure builds up, it balloons out at its weakest point. Aneurysms that occur in the brain are termed as Cerebral Aneurysm (CA). In early stage of aneurysm development it goes unnoticed, with minor to no symptoms at all. As the aneurysm develops in size, the symptoms get prominent. Symptoms like numbness in the face, or problems with the eyes. Aneurysm exceeding the $25 \mathrm{~mm}$ diameter value is termed as giant aneurysm. At this stage it demands immediate surgical attention, to prevent cerebral aneurysm ruptures or subarachnoid haemorrhage (SAH). In this stage the symptoms get worse and might include nausea, vomiting, eyesight problems, seizures (fits), loss of consciousness and even death. Recent statistics indicate sudden rise in SAH cases (1 cases of SAH/year/10,000 people). Apart from having single cerebral aneurysm; multiple cerebral aneurysms also exist in practice. Multiple cerebral aneurysms occur in group with an incidence rate of $20-30 \%$ [9].

Most cerebral aneurysms develop at the branches in blood vessels because the walls in these sections are weaker. Cerebral aneurysm mainly develops due to weakness in the artery wall present since birth, high blood pressure due to hypertension, and arteriosclerosis. Cerebral Aneurysm diagnosis includes CT (computerized tomography) scan, cerebrospinal fluid test, MRI (magnetic resonance imaging) scan and Cerebral arteriogram (cerebral angiogram). Patients diagnosed with ruptured cerebral aneurysm got two options; surgical clipping or endovascular coiling. If detected at an early stage, SAH can be prevented by keeping under control blood pressure and blood cholesterol level. 


\section{Digital subtraction angiography (DSA)}

Digital subtraction angiography (DSA) is a type of fluoroscopic technique used by radiologist to clearly visualize the blood vessels from the background of bony or dense soft tissue environment by controlled administration of radio-opaque contrast region [10]. In principle, it subtracts a post-opacification image called the 'Live' image from a pre-opacification image of the same region called the 'Mask' image and hence the name. During this $\mathrm{X}$-ray test, a catheter is inserted through a blood vessel in the groin or arm and guided up through the vessel into the brain, through which radio-opaque dye is then injected into the cerebral artery. Although this test is more invasive and carries more risk than the other tests, still it is the best way or gold standard investigation [11] to locate small (less than $5 \mathrm{~mm}$ ) brain aneurysms. This test can help to diagnose such conditions as the presence of a blood clot, fatty plaque that increases the patient's risk of stroke, cerebral aneurysm [7] or other vascular malformations.

Consider a patient with anatomical thickness of $x_{t}$, arterial thickness of $x_{l}$ and a linear attenuation coefficient of $u_{t}$. Then the intensity of the pre-opacification image or 'Mask' image and the post-opacification image or 'Live' image is given by $I_{m}$ and $I_{I}$ respectively in Eq. (1), where $I_{0}$ represents the photon fluence delivered to the image intensifier without any opacifying agent.

$$
\begin{aligned}
I_{m} & =I_{0} e^{-u_{t} x_{t}} \\
I_{I} & =I_{0} e^{-\left(u_{t} x_{t}+u_{I} x_{I}\right)}
\end{aligned}
$$

The DSA is then obtained by logarithmic subtraction [12] of post-opacification image from pre-opacification image.The logarithmically subtracted image $S_{l o g}$ is therefore depicted as:

$$
\begin{aligned}
S_{l o g} & =\ln I_{m}^{\prime}-\ln I_{I}^{\prime} \\
& =\left[-u_{t} x_{t}\right]-\left[-\left(u_{t} x_{t}+u_{I} x_{I}\right)\right] \\
& =u_{I} x_{I}
\end{aligned}
$$

\section{Auto-Threshold}

Thresholding is a method of binary image segmentation [13]. It is a fundamental step for successful CA detection [14]. In this method we will determine optimum Global Threshold value to partition the CA image into a meaningful region.

\subsection{Conversion of RGB DICOM image to grayscale image}

Angiography has traditionally used analog media for long term storage. But to meet the demand of digital media, the National Electrical Manufacturers' Association and various professional organizations from around the world have collaborated to develop the DICOM (Digital Imaging and Communications in Medicine) image formatting standard to ensure global intra operability of medical instrumentation [15].

In our experiment we have used color enhanced Cerebral Angiogram which uses RGB color space. It is converted into grayscale image $(\mathrm{Y})$ by evaluating the luminance of the color image abiding MPEG standard using Eq. (3).

$$
Y=0.299 R+0.587 G+0.114 B
$$

The coefficients represent human perception of colors, in particular that humans are more sensitive to green and least sensitive to blue. 


\subsection{Vessel Enhancement using Gaussian Filtering}

Inspired from the works of Frangi et al. [16] and Li et al. [17] where the vessel radius is estimated from the deviation of the smoothing Gaussian kernel, here Gaussian filtering is employed as an important pre-processing step. It reduces the background noise and enhances the vessel structures.

For an image size $M \times N$, variables $\mathrm{x}$ and y represent position of pixel in x-direction and y-direction respectively and given by eq. (4)

$$
\begin{aligned}
& x \in X \text { where } X=1,2, \ldots, N \\
& y \in Y \text { where } Y=1,2, \ldots, M
\end{aligned}
$$

In general, linear spatial filtering of an image $(Y)$ of size $M \times N$ with a filter $(f)$ of mask size $m \times m$ is given by the expression :

$$
F(x, y)=\sum_{s=-a}^{a} \sum_{t=-a}^{a} Y(x, y) f(x+s, y+t)
$$

where,

$$
\begin{aligned}
m & =2 a+1 \\
f(x, y) & =\frac{1}{\sqrt{2 \pi} \sigma^{2}} e^{-\frac{x^{2}+y^{2}}{2 \sigma^{2}}}
\end{aligned}
$$

In our experiment we have used an isotropic (i.e., circularly symmetric) Gaussian filter function with distribution of mean $(0,0)$ and $\sigma=$ Standard deviation of Image $(Y)=\sqrt{\mathbf{E}\left[Y^{2}\right]-(\mathbf{E}[Y])^{2}}$ having convolution kernels of size $3 \times 3$ (i.e., $\mathrm{m}=3$ ). Using kernel size greater than 3 leads to over-estimation of aneurysm shape in section 5.4 of the algorithm.

\subsection{Finding image gradient using two spin operator}

Two spin operator is a $3 \times 3$ neighborhood based gradient operator. The convolution masks for the two spin operator are defined by the two kernels as shown in Table 1 .

Table 1: Convolution masks for two spin operator

(a) Mask 1

(b) Mask 2

\begin{tabular}{|c|c|c|}
\hline 1 & 0 & 0 \\
\hline 1 & -4 & 1 \\
\hline 0 & 0 & 1 \\
\hline
\end{tabular}

\begin{tabular}{|c|c|c|}
\hline 0 & 1 & 1 \\
\hline 0 & -4 & 0 \\
\hline 1 & 1 & 0 \\
\hline
\end{tabular}

These two masks are separately applied on the input image to yield two gradient components $G_{1}$ and $G_{2}$ in the horizontal and vertical orientations respectively. It is a kind of laplacian impulse response kernel.

$$
\begin{aligned}
& G_{1}(x, y)=[F(x-1, y-1)+F(x-1, y)+F(x+1, y)+F(x+1, y+1)]-4[F(x, y)], \forall x, y \in F \\
& G_{2}(x, y)=[F(x+1, y-1)+F(x, y-1)+F(x, y+1)+F(x-1, y+1)]-4[F(x, y)], \forall x, y \in F
\end{aligned}
$$

The gradient magnitude is computed as

$$
G(x, y)=\sqrt{G_{1}^{2}(x, y)+G_{2}^{2}(x, y)}
$$


As can be clearly seen in Table 1 two orthogonal masks are superimposed to give arise to double spin like characteristics. It has got four arms bent at right angles with $90^{\circ}$ rotational symmetry and chirality, hence the absence of reflectional symmetry. It enhances the convex aneurysms and reduces the cylindrical vessel structures. Maximum of the Gradient magnitude measures the standard deviation of the image with special emphasis to rotationally symmetric vessels.

\subsection{Thresholding}

The threshold for the image is evaluated as

$$
I(x, y)= \begin{cases}1 & : F(x, y)<\lambda \\ 0 & : F(x, y) \geq \lambda\end{cases}
$$

where

$$
\lambda=\min [\underset{(x, y) \in(X \times Y)}{\forall} F(x, y)]+\max [\underset{(x, y) \in(X \times Y)}{\forall} G(x, y)]
$$

\subsection{Performance of Auto-Threshold}

Auto-Threshold of binary image of vessel structures from the grayscale DICOM image, is compared against two most widely cited thresholding algorithms, i.e. Otsu's threshold[18] and Sauvola's threshold[19]. Sauvola's threshold [19] is the most widely cited local adaptive thresholding with local binarization technique benchmark of $94.9 \%$. While, Otsu's method [18] is the most widely cited global thresholding technique.

The performance section consists of two comparison results :

Figure 1 shows the visual comparison of the results of 3 threshold methods, viz.: Sauvola's Threshold, Otsu's Threshold and Auto-Threshold.

Figure 2 shows the comparison of performance of 2 global threshold methods, viz.: Auto-Threshold method and Otsu's Threshold method. Graphs are plotted for each test images for the parameters Sensitivity, Specificity and Accuracy against Global Threshold value which varies from 0 to 255. Otsu's Threshold and AutoThreshold value are marked in each graph in red and green dotted lines respectively. Details of the parameters are defined in section 7 .

[I] For 1st Test Image

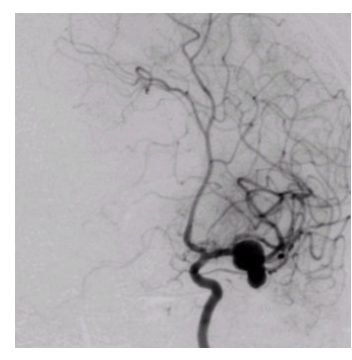

(a) Original Image

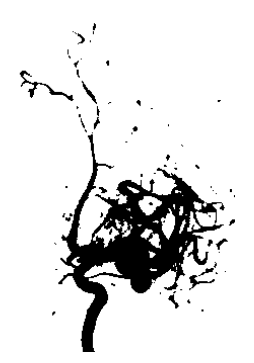

(b) Sauvola's Threshold

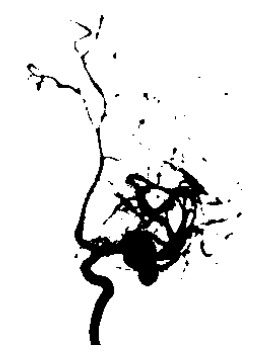

(c) Otsu's Threshold

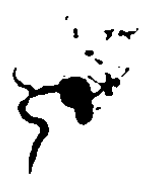

(d) Auto-Threshold 
[II] For 2nd Test Image

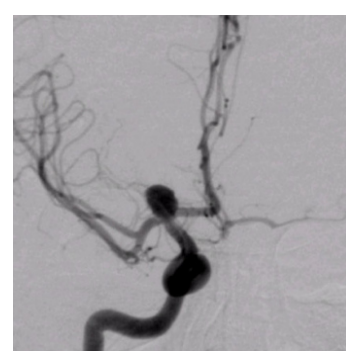

(a) Original Image

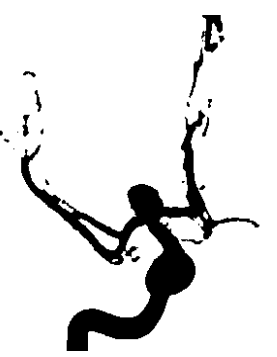

(b) Sauvola's Threshold

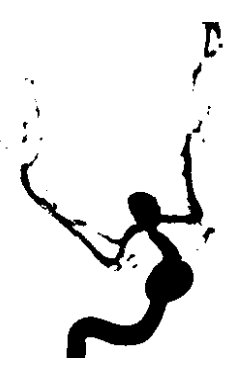

(c) Otsu's Threshold

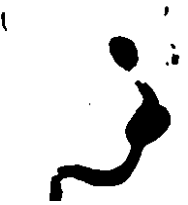

(d) Auto-Threshold

[III] For 3rd Test Image

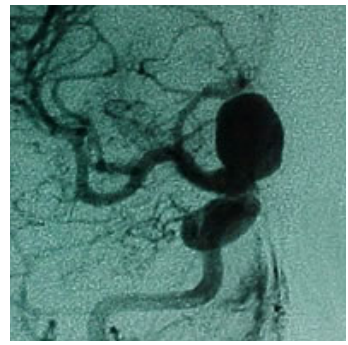

(a) Original Image

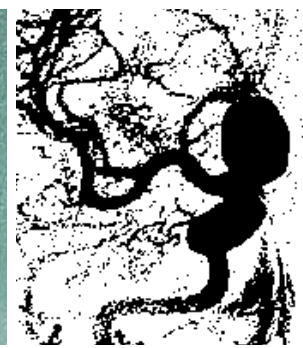

(b) Sauvola's Threshold

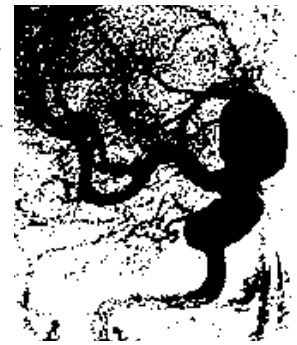

(c) Otsu's Threshold

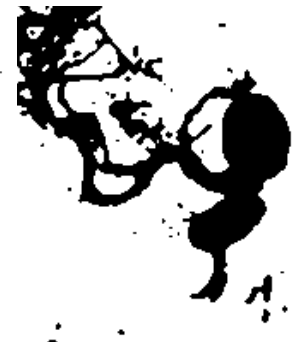

(d) Auto-Threshold

[IV] For 4th Test Image

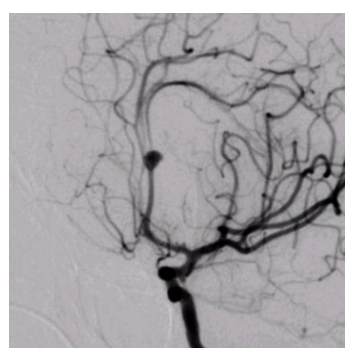

(a) Original Image

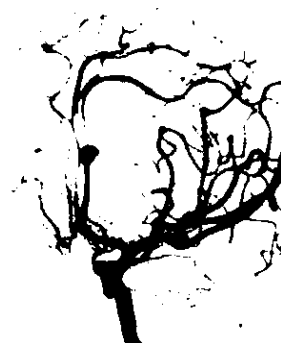

(b) Sauvola's Threshold

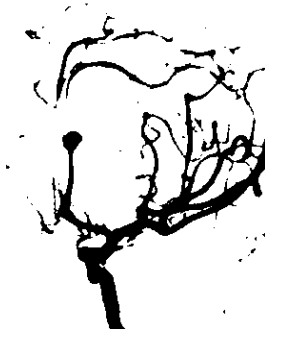

(c) Otsu's Threshold

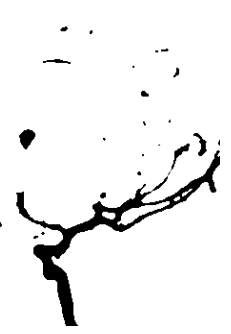

(d) Auto-Threshold 


\section{[V] For 5th Test Image}

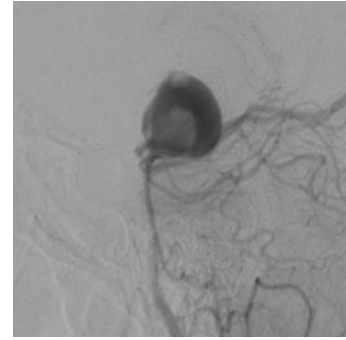

(a) Original Image

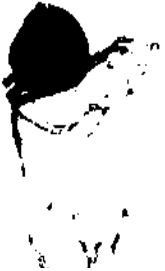

(b) Sauvola's Threshold

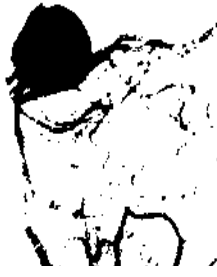

(c) Otsu's Threshold

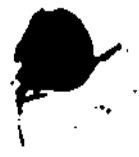

? 1

Figure 1: Results of the three thresholding methods (a)Sauvola's Threshold[19]; (b)Otsu's Threshold[18]; (c)Auto-Threshold over the original grayscale 5 test images

[I] For 1st Test Image

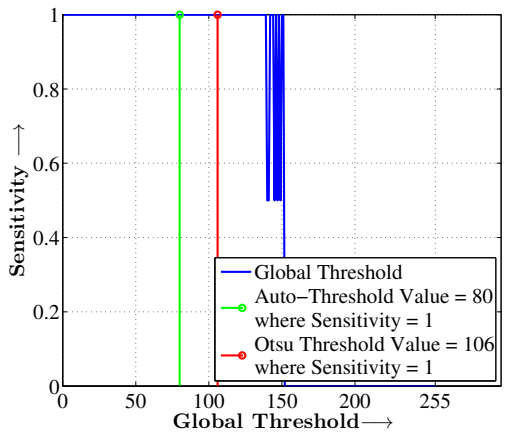

(a)

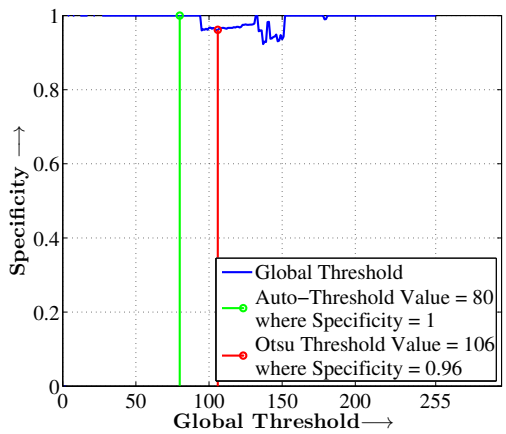

(b)

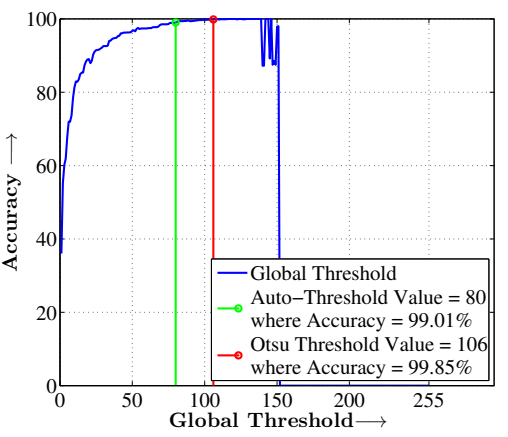

(c)
[II] For 2nd Test Image

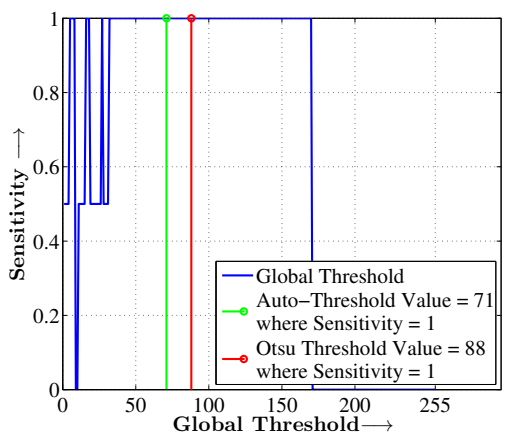

(a)

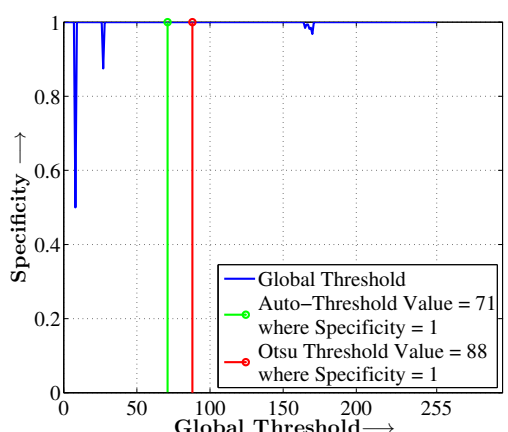

(b)

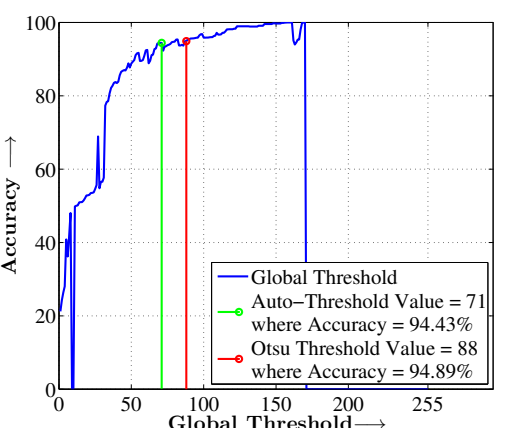

(c) 
[III] For 3rd Test Image

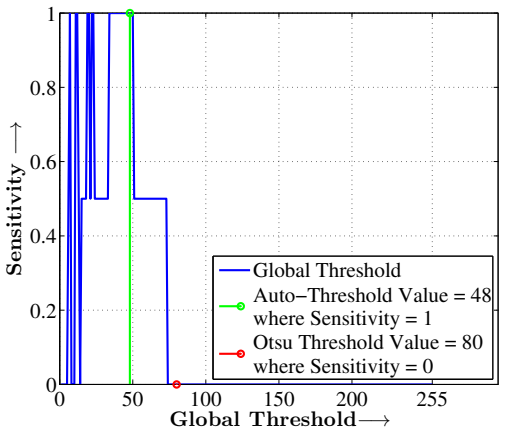

(a)

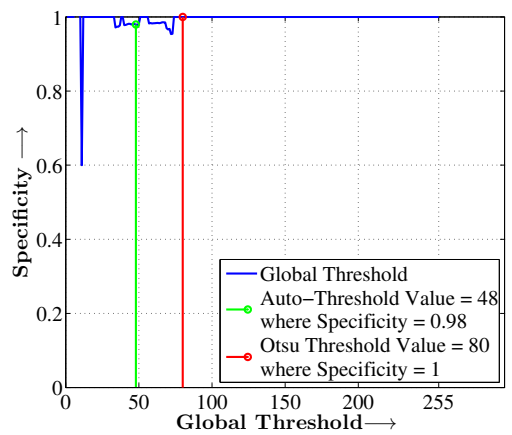

(b)

\section{[IV] For 4th Test Image}

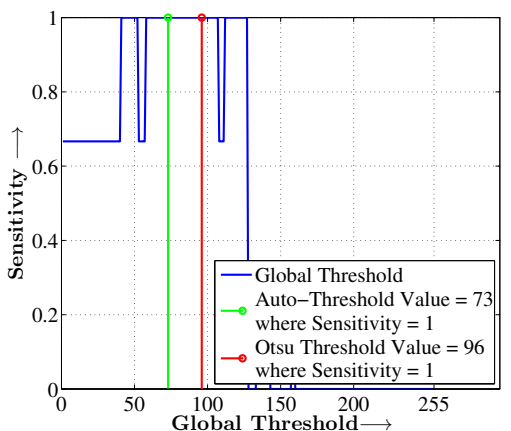

(a)

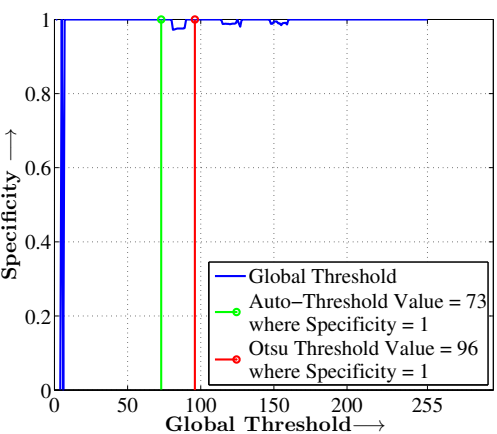

(b)

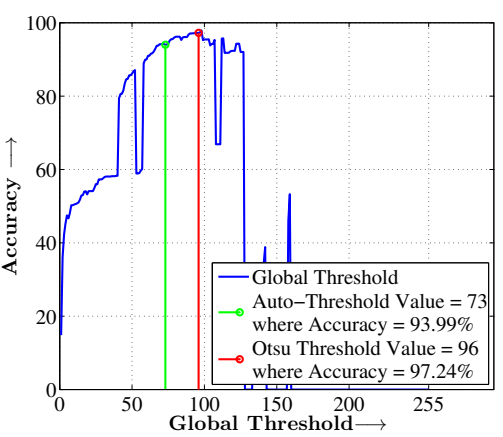

(c)

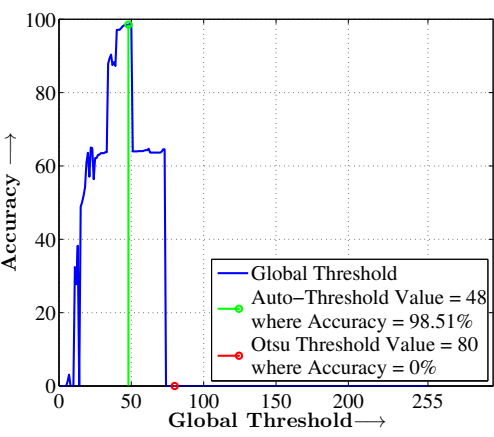

(c)

\section{[V] For 5th Test Image}

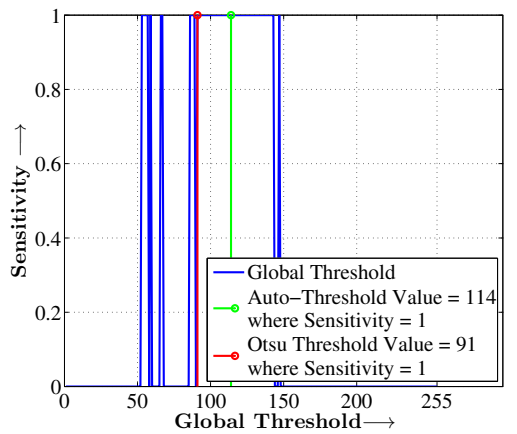

(a)

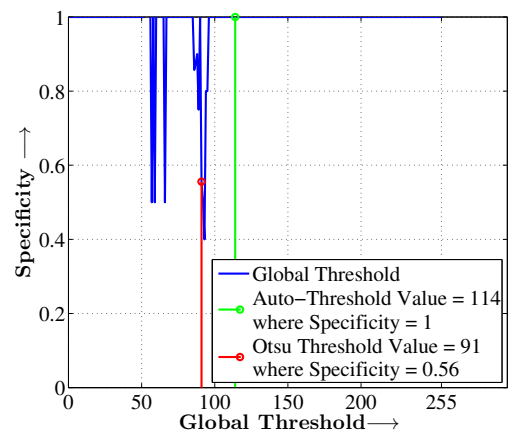

(b)

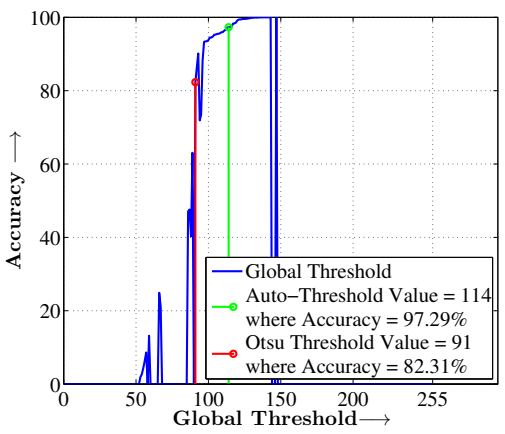

(c)

Figure 2: Comparison of performance of Auto-Threshold method with Otsu's Threshold for 5 test images on the basis of parameter (a) Sensitivity, (b)Specificity and (c)Accuracy against Global Threshold value [0 255]

From the above figures it can be concluded that the Auto-Threshold outperforms other 2 thresholding methods in its segmentation of aneurysms along with major vessels while suppressing the minor vessels. 


\section{Modified Hough Circle Transform \& Peak Trekking (MHCT-PT) technique}

\subsection{Hough Transform (HT)}

Hough Transform (HT) [20, 21] is a template matching technique that locates shapes in images. It has found extensive applications in extracting lines, circles, ellipses and other conic sections of interest [22]. HT computation requires a mapping from the image points into an accumulator space or Hough space. This mapping function should be computationally efficient for reduction of time complexity. Although it requires significant amount of space for storage, but it is immaterial when compared with its shape extraction capabilities. Ecabert et al. [23] in their works asserted that the generalized Hough Transform is valid for developing a method which works without human intervention and for different medical image perspective. Hough transform for circles is chosen as our preferred method of shape extraction [24, 25], since aneurysms are in general of saccular or balloon type. Though this method is less popular in the literature, but its ability to detect bulging region of aneurysms is unparallel.

\subsection{Drawing circle in discrete space}

\subsubsection{Midpoint Circle Algorithm}

Midpoint Circle Algorithm [26, 27] has been proved to be one of the most efficient algorithms to calculate the pixel positions around a circular path centred at the coordinate origin $(0,0)$ with a given radius $r$. This generated circle can then be shifted to proper screen position by moving its centre to $\left(x_{c}, y_{c}\right)$. Because of its regularity, a circular section can be broken down into an eight point symmetric region, as drawn in Figure 3 . The circle section from the line $y=0$ to $y=x$ forms the first octant.

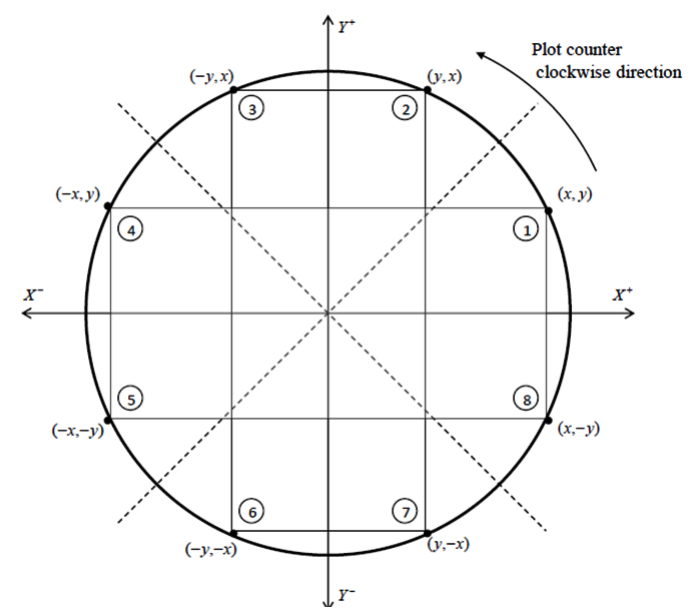

Figure 3: Eight-point symmetry of a circular region

In the 1 st octant the slope of the curve varies from 0 to 1 . So unit steps can be taken in the positive $x$ direction over this octant and a decision parameter to predict the proximity of the two possible $y$ positions to the circle path at each step. A circle function $f_{\text {circle }}(x, y)$ is therefore defined in this connection which can be applied in this method.

$$
f_{\text {circle }}(x, y)=x^{2}+y^{2}-r^{2}
$$

Relative position of any point $(x, y)$ with respect to the circle boundary can be determined by checking the polarity of the circle function as illustrated below : 


$$
f_{\text {circle }}(x, y) \text { is } \begin{cases}<0 & : \text { if }(x, y) \text { is inside the circle boundary } \\ =0 & : \text { if }(x, y) \text { is on the circle boundary } \\ >0 & : \text { if }(x, y) \text { is outside the circle boundary }\end{cases}
$$

Assuming that pixel $\left(x_{k}, y_{k}\right)$ has just been plotted, the immediate next pixel to plot has to be selected by the decision parameter $\left(p_{k}\right)$ from the two pixel positions i.e. $\left(x_{k}+1, y_{k}\right)$ and $\left(x_{k}+1, y_{k}-1\right)$. The decision parameter $\left(p_{k}\right)$ is nothing but the circle function evaluated at the midpoint between these two pixels.

$$
\begin{aligned}
p_{k} & =f_{\text {circle }}\left(x_{k}+1, y_{k}-\frac{1}{2}\right) \\
& =\left(x_{k}+1\right)^{2}+\left(y_{k}-\frac{1}{2}\right)^{2}-r^{2}
\end{aligned}
$$

The next pixel $\left(x_{k+1}, y_{k+1}\right)$ to be chosen depends on the sign of $p_{k}$, as shown below :

$$
\begin{aligned}
& x_{k+1}=x_{k}+1 \\
& y_{k+1}= \begin{cases}y_{k} & \text { when } p_{k}<0 \\
y_{k}-1 & \text { when } p_{k} \geq 0\end{cases}
\end{aligned}
$$

Successive decision parameters $\left(p_{k+1}\right)$ can easily be obtained by using some incremental calculations without evaluating the circle function repeatedly. This saves computation time tremendously. A recursive expression is shown below for clarity during the calculation of the next decision parameter from the present decision parameters.

$$
\begin{aligned}
p_{k+1} & =f_{\text {circle }}\left(x_{k+1}+1, y_{k+1}-\frac{1}{2}\right) \\
& =\left(\left(x_{k}+1\right)+1\right)^{2}+\left(y_{k+1}-\frac{1}{2}\right)^{2}-r^{2} \\
& =p_{k}+2\left(x_{k}+1\right)+\left(y_{k+1}^{2}-y_{k}^{2}\right)-\left(y_{k+1}-y_{k}\right)+1 \\
\text { or, } \quad p_{k+1} & = \begin{cases}p_{k}+2 x_{k+1}+1 & \text { when } p_{k}<0 \\
p_{k}+2 x_{k+1}-2 y_{k+1}+1 & \text { when } p_{k} \geq 0\end{cases}
\end{aligned}
$$

The initial decision parameter is chosen by evaluating the circle function at the start position $\left(x_{0}, y_{0}\right) \equiv(0, r)$ given by Eq. 144 :

$$
\begin{aligned}
p_{0} & =f_{\text {circle }}\left(1, r-\frac{1}{2}\right) \\
& =1+\left(r-\frac{1}{2}\right)^{2}-r^{2} \\
& =\left(\frac{5}{4}-r\right)
\end{aligned}
$$




\subsection{Modified Hough Circle Transform (MHCT)}

It is a mapping function that maps binary image points in $I$ to a 3D parameter space defined as Hough Hierarchy $(\mathbb{H})$.

Prior to the beginning of introduction to Hough Hierarchy, we need to introduce few mathematical notations.

$$
f_{\text {circle }}^{r}\left(x-x_{c}, y-y_{c}\right)=\left(x-x_{c}\right)^{2}+\left(y-y_{c}\right)^{2}-r^{2}
$$

where $f_{\text {circle }}^{r}\left(x-x_{c}, y-y_{c}\right)$ is a circle function with centre at point $\left(x_{c}, y_{c}\right)$ having radius $r$, followed from eq. (9).

Points lying on the boundary of the circle function is given by eq. (16) while those lying interior to the annular region bounded by the radius $r_{1}$ and $r_{2}$ is given by eq. (17)

$$
\begin{aligned}
& \delta \Omega_{r}\left(x_{c}, y_{c}\right)=\left\{(x, y) \in(X \times Y): f_{\text {circle }}^{r}\left(x-x_{c}, y-y_{c}\right)=0\right\} \\
& { }_{r_{1}} \Omega_{r_{2}}^{-}\left(x_{c}, y_{c}\right)=\left\{(x, y) \in(X \times Y): f_{\text {circle }}^{r_{1}}\left(x-x_{c}, y-y_{c}\right)>0 \wedge f_{\text {circle }}^{r_{2}}\left(x-x_{c}, y-y_{c}\right)<0\right. \text {, } \\
& \left.0 \leq r_{1} \leq r_{2}\right\}
\end{aligned}
$$

where '-' sign in $r_{1} \Omega_{r_{2}}^{-}$indicates that points lying within the interior of the annular region instead of exterior to it.

The mapping function for our MHCT algorithm is defined by the eq. (18), which is also attributed as Votes casted at co-ordinate point $(x, y)$

$$
v(x, y)=\sum_{r=1}^{\substack{\text { Hierarchy } \\ \text { Height }}}\left\lfloor\frac{\left|\delta \Omega_{r}(x, y) \cap I\right|}{\left|\delta \Omega_{r}(x, y)\right|}\right\rfloor
$$

where operator $|$.$| means cardinality of the set, here it is used to find number of pixels and operator \lfloor$.$\rfloor is used$ as floor operator.

Hough Hierarchy, is generated by the accumulation of the casted votes for all pixel positions within the image, given by the relation in eq. (19). It is illustrated in fig. 4 .

$$
\mathbb{H}=\left\{(x, y) \in(X \times Y): \bigcup_{\forall(x, y)} v(x, y)\right\}
$$

The co-domain the function $v(x, y)$ and upper limit of relation $\mathbb{H}$ is given in the eqs. (20) and (21) respectively.

$$
\begin{aligned}
V & =\{0,1,2, \ldots, \text { Hierarchy Height }\}, \text { where } v(x, y) \in V \\
\max (\mathbb{H}) & =\{(x, y) \in X \times Y, v(x, y) \in \mathbb{H}: \max [v(x, y)]\}
\end{aligned}
$$

Hough Hierarchy formally is a function that associates to every pair $(x, y)$ in $X \times Y$ an element $v(x, y)$ in $\mathrm{V}$, as shown in eq. 22). Therefore its graph consists of pairs of the form $(x, y, v(x, y))$. Such pairs in which the first element is itself a pair are often identified with triples. This makes the graph of $\mathbb{H}$ a ternary relation between X, Y and V, as represented in eq. 23]. Hence, Hough Hierarchy is a 3D parameter space, as shown by the mesh diagram in figures $12 \mathrm{e}$ ) to $16 \mathrm{e}$.

$$
\begin{gathered}
\mathbb{H}: X \times Y \rightarrow V \\
X \times Y \times V=\{(x, y, v(x, y)): x \in X \wedge y \in Y \wedge v(x, y) \in V\}
\end{gathered}
$$




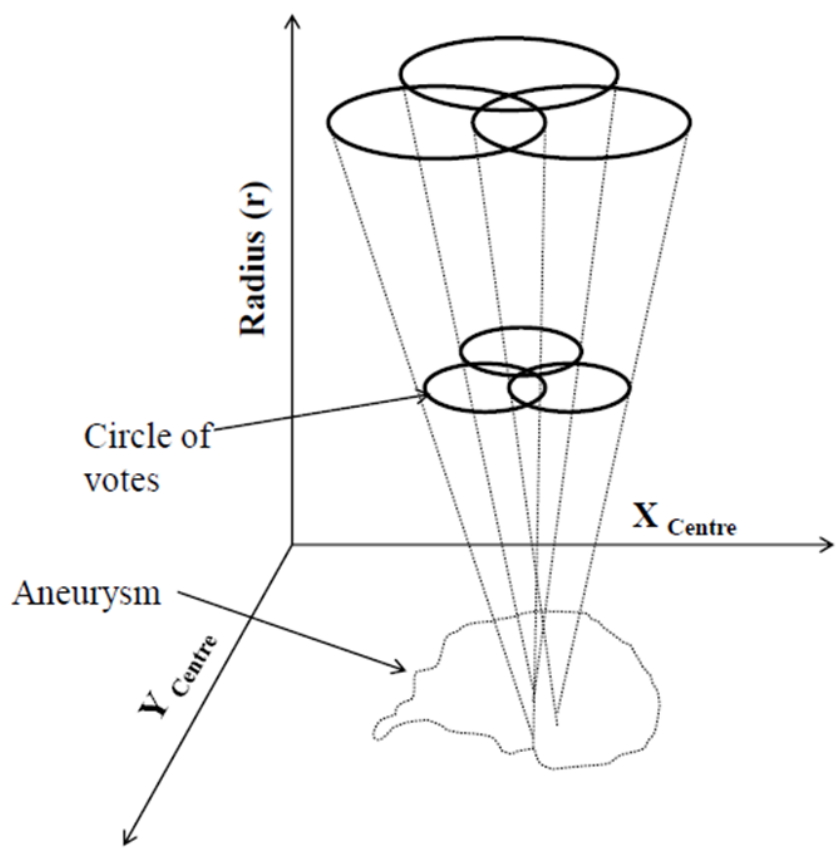

Figure 4: 3D parameter space of Hough hierarchy

\subsection{Peak Trekking}

Since, Hough Hierarchy 3D parameter space resembles a mountain range, it can be explored like a mountain expedition. Hence the name of this novel algorithm is chosen as Peak Trekking. This proposed algorithm acts like a reverse mapping of a 3D local mountain from the mountain range (i.e, Hough Hierarchy, 3D parameter space) to a $2 \mathrm{D}$ binary detected regions.

Let, the outer and inner radius of an annular region be defined by the variable $r_{\text {out }}$ and $r_{\text {in }}$ in eqs. (24) and (25) respectively.

$$
\begin{aligned}
r_{\text {out }} & =\max (\mathbb{H}) \\
r_{\text {in }} & =r_{\text {out }}-\text { Neighborhood Depth }
\end{aligned}
$$

where parameter Neighborhood Depth indicates the width of the annular region. It is an important parameter as it controls over-segmentation or under-segmentation of the detected aneurysm regions.

The range of the variable $r_{\text {in }} \& r_{\text {out }}$ lies within $[0, \max (\mathbb{H})]$.

Initial step of the Peak Trekking algorithm is to get the position of the highest peak from the 3D parameter space and stores it in the first position of the array $\mathrm{C}$.

$$
\mathrm{C}[1]=\{(x, y) \in(X \times Y): \mathbb{H}(x, y) \cap \max (\mathbb{H}) \wedge|\mathbb{H}(x, y) \cap \max (\mathbb{H})|=1\}
$$

where condition $|\mathbb{H}(x, y) \cap \max (\mathbb{H})|=1$ makes it clear that even if many pixels might satisfy the condition in eq. (26), but only one pixel will be considered.

A circular region with centre at $\mathrm{C}[1]$ having radius $r_{\text {out }}$ is drawn using eq. 27]

$$
\mathrm{R}_{1}=\left\{(x, y) \in(X \times Y):{ }_{0} \Omega_{r_{\text {out }}}^{-}(\mathrm{C}[1])\right\}
$$

Next step is to find the pixel positions that lie within the annular region with centre at $\mathrm{C}[1]$ bounded by the inner and outer radius of $\left(r_{i n}-1\right)$ and $\left(r_{\text {out }}-1\right)$ respectively. These positions evaluated in eq. (28) are stored 
in 2 nd position of the array C. Here, the boundary radii are reduced by ' 1 ' to indicate a unit decrement in level along dimension $V$ of the 3D parameter space.

$$
\mathrm{C}[2]=\left\{(x, y) \in(X \times Y): r_{\text {in }-1} \Omega_{r_{\text {out }}-1}^{-}(\mathrm{C}[1])\right\}
$$

The pixel positions stored in $\mathrm{C}[2]$ in eq. 28, are treated as centres for generating circular regions in eq. 29] with uniform radius $\left(r_{\text {out }}-1\right)$.

$$
\mathrm{R}_{2}=\left\{(x, y) \in(X \times Y):{ }_{0} \Omega_{r_{\text {out }}-1}^{-}(\mathrm{C}[2])\right\}
$$

By principle of mathematical induction, the eqs. 26) to (29) can be generalised by the recursive relation as in eqs. 30 and 31 . Except for level $1(n=1)$, where $C[1]$ is defined by eq. 26 .

$$
\begin{aligned}
\mathrm{C}[n] & = \begin{cases}\left\{(x, y) \in(X \times Y):{ }_{\left[r_{\text {in }}-(n-1)\right]} \Omega_{\left[r_{\text {out }}-(n-1)\right]}^{-}(\mathrm{C}[n-1])\right\} & : n>1 \\
\{(x, y) \in(X \times Y): \mathbb{H}(x, y) \cap \max (\mathbb{H}) \wedge|\mathbb{H}(x, y) \cap \max (\mathbb{H})|=1\} & : n=1\end{cases} \\
\mathrm{R}_{n} & =\left\{(x, y) \in(X \times Y):{ }_{0} \Omega_{\left[r_{\text {out }}-(n-1)\right]}^{-}(\mathrm{C}[n])\right\}
\end{aligned}
$$

where $n$ gives the level number, as $n \in V$

The $\mathrm{n}$ circular regions generated from eq. (31) are superimposed to give the 1st detected region $\left(\mathrm{D}_{1}\right)$, shown in eq. (32). This mathematical operation has been illustrated in the fig. 5 .

$$
\mathrm{D}_{1}=\bigcup_{n=1}^{\substack{\max (\mathbb{H}) \\-\text { Neighborhood } \\ \text { Depth }}} \mathrm{R}_{n}
$$

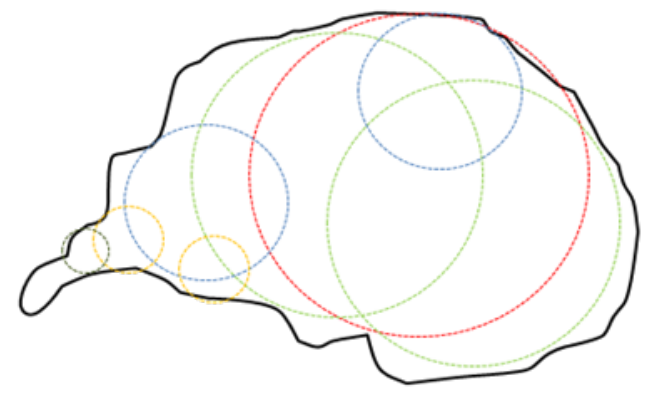

Figure 5: Schematic diagram of the detected region

where,uniform radius are marked by same color viz., red:level-1; green:level-2; blue:level-3; yellow:level-4; black:level-5

The 1st detected region $\left(D_{1}\right)$ is now subtracted from the Hough Hierarchy, 3D parameter space to prevent redundancy in detected region. This relation is shown in eq. 33.

$$
\mathbb{H}=\mathbb{H} \cap \overline{\mathrm{D}}_{1}
$$

This process of region detection and region subtraction is iterated till the condition $\max (\mathbb{H}) \geq$ Peak Depth is violated, shown in eq. (34). Parameter Peak Depth decides whether the height of the post processed Hough Hierarchy, 3D parameter space is sufficient for the regions being called as mountain any more, or it has become a valley. Practically, Peak Number acts as a filter for noisy shape.

$$
\mathbb{H}=\mathbb{H} \cap \overline{\mathrm{D}}_{n_{p}}: \max (\mathbb{H}) \geq \text { Peak Depth }
$$


where $n_{p}$ denotes the peak number, shown in eq. (35).

Peak Number $\left(n_{p}\right)$ is like a unique ID for the detected regions. It maintains the index of the detected regions as if arranged in a queue, in order to maintain the principle of First In First Out (FIFO) to retain the hierarchy information. Thus, initially detected regions have lower Peak Number $\left(n_{p}\right)$, than latter.

$$
n_{p} \in N_{p}, N_{p}=\left\{1,2, . . n_{p}^{\max }\right\}
$$

where $n_{p}^{\max }$ is the total number of detected regions generated.

Finally, the detected regions are given by the relation in eq. (36)

$$
\mathbb{D}=\left\{n_{p} \in N_{p}: \mathrm{D}_{n_{p}}\right\}
$$

\subsection{Aneurysm detection}

Cerebral aneurysms are commonly described as balloon-like bulge of a blood vessel localized on an artery trajectory. It is roughly spherical having width greater than the neighboring parent vessels. Radiologist mainly uses circularity and diameter size as important features for aneurysm detection. In our experiment we have taken into consideration three features, defined by the following three parameters.

\subsubsection{Parameters}

Peak number $\left(\mathbf{n}_{\mathbf{p}}\right)$ Peak number indicates the convexity of a shape. Higher the peak number more convex or spread out the shape appears.

Area $\left(A_{\mathbb{D}_{n_{p}}}\right) \quad$ The area of the detected shape for a particular peak number $\left(n_{p}\right)$ is not calculated geometrically but by counting the number of pixels within the shape. Though we have used pixel area, but it can be correlated with the actual area by multiplying with the image scaling factor.

$$
A_{\mathbb{D}_{n_{p}}}=\sum_{\forall(x, y)} D_{n_{p}}
$$

Compactness Factor $\left(C_{\mathbb{D}_{n_{p}}}\right) \quad$ It is defined as the measure of the circularity of a shape [28], given by the equation Eq. (38):

$$
C_{\mathbb{D}_{n_{p}}}=\frac{4 \pi A_{\mathbb{D}_{n_{p}}}}{P_{\mathbb{D}_{n_{p}}}^{2}}
$$

where ' $A_{\mathbb{D}_{n_{p}}}$ ' and ' $P_{\mathbb{D}_{n_{p}}}$ ' indicate area and perimeter of the detected shape respectively for a particular peak number.

If compactness factor is close to 1 then it is more or less circular, but if it is close to 0 then it indicates a tubular or a rectangular structure. This parameter will help to distinguish between normal and bulged vessels.

\subsubsection{Boundary conditions}

Queue of the generated shapes might contain aneurysms of different sizes, arteries, vessels, angiographic stain and even background noises. So, it is necessary to isolate aneurysm portion from other detected shapes. This detection of aneurysm depends upon three conditions as described by eqs. (39) to 41).

\section{Condition 1.}

$$
A_{\mathbb{D}_{n_{p}}} \geq \text { Minimumarea }
$$


where

$$
\text { Minimumarea }=100 \times\left\lfloor\frac{A_{\mathbb{D}_{1}}}{100 \times \mathbf{k}}\right\rfloor
$$

where $A_{\mathbb{D}_{1}}$ represents area of the shape $\left(\mathbb{D}_{1}\right)$ generated by peak number 1 , i.e $\left(n_{p}=1\right)$.

\section{Condition 2.}

$$
C_{\mathbb{D}_{n_{p}}} \geq \mathbf{C}
$$

where $k, C$ are optimized weights derived experimentally.

Condition 3. True for shape $\mathbb{D}_{n_{p}}$, if conditions 1,2 and 3 are evaluated to be true for shape $\mathbb{D}_{n_{p}-1}$. This condition is always true for shape $\mathbb{D}_{n_{1}}$.

Here, conditions 1 and 2 are necessary for aneurysm detection. But condition 3 is necessary and sufficient for our algorithm in aneurysm detection process to terminate.

\subsubsection{Aneurysm region detection}

Eq. (42) gives the relation of how Aneurysm regions are identified from the detected regions.

$$
\mathbb{A}=\left\{n_{p} \in N_{p}: \mathrm{D}_{n_{p}}\right\} \text { where } \mathrm{D}_{n_{p}} \text { is subject to conditions } 1,2 \& 3
$$

\subsection{Aneurysm Classification}

The severity of an aneurysm can be classified on the basis of its diameter [29]. The corresponding relationship with diameter is given by the eq. (44) :

$$
\operatorname{Diameter}\left(D_{\mathbb{D}_{n_{p}}}\right)=2 \sqrt{\frac{A_{\mathbb{D}_{n_{p}}}}{\pi}} \times(\text { Scaling Factor })
$$

where Scaling Factor is the link between relative DSA output measurements to the vessel's absolute measurements.

$$
\text { Aneurysm } \equiv \begin{cases}\text { Small } & : D_{\mathbb{D}_{n_{p}}}<7 \mathrm{~mm} \\ \text { Medium } & : 7 \mathrm{~mm} \leq D_{\mathbb{D}_{n_{p}}}<15 \mathrm{~mm} \\ \text { Large } & : 15 \mathrm{~mm} \leq D_{\mathbb{D}_{n_{p}}}<25 \mathrm{~mm} \\ \text { Giant } & : 25 \mathrm{~mm} \leq D_{\mathbb{D}_{n_{p}}}<50 \mathrm{~mm} \\ \text { SuperGiant } & : D_{\mathbb{D}_{n_{p}}} \geq 50 \mathrm{~mm}\end{cases}
$$

\section{Proposed algorithm}

In this communication, we have proposed three novel algorithms: Auto-thresholding, using two-spin operator; Modified Hough Circle Transform \& Peak trekking (MHCT-PT) technique and classification of cerebral aneurysm on the basis of its severity. Algorithm 1 briefly summarizes this entire process. 


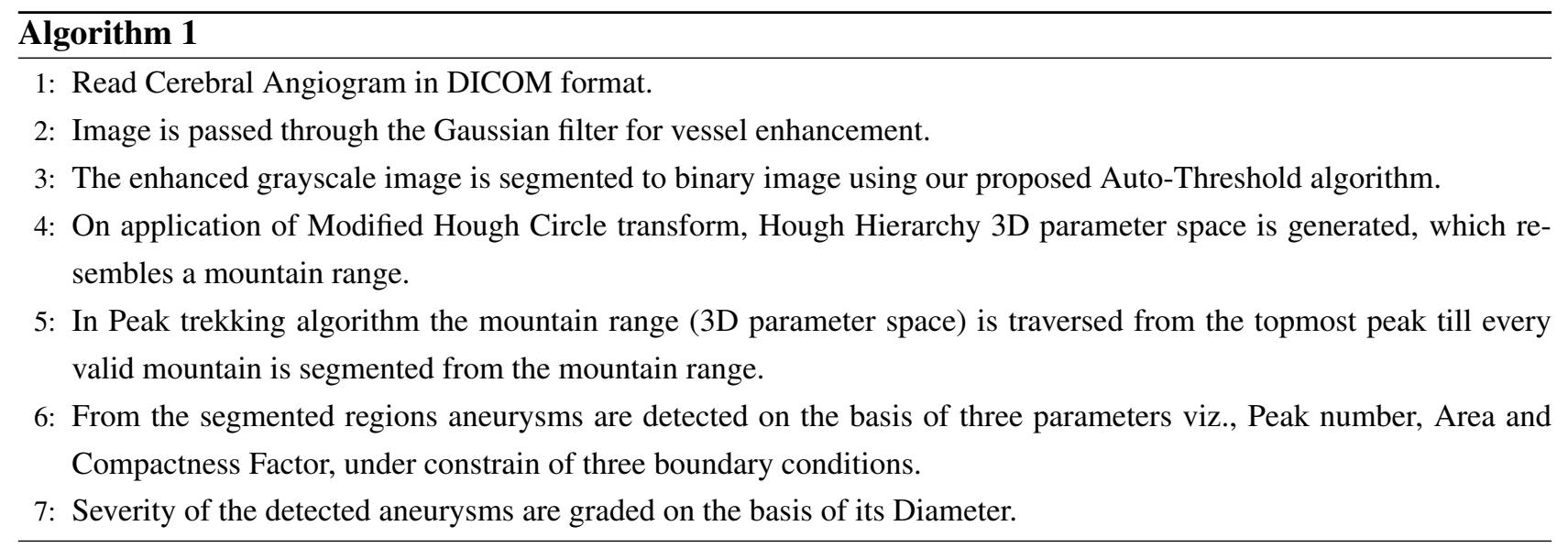

\section{Parameter optimization}

Since we wished to conduct quantitative studies of aneurysm, we first carried out an analysis of our aneurysm segmentation algorithm and the effect of various algorithmic parameters on this accuracy. Adjustable parameters in our algorithm include: (a) Hierarchy Height; (b) Neighborhood Depth; (c) Peak Depth; (d) C; (e) $k$;

This is a problem of multi-parameter optimization. Of these the significant parameter turns out to be Neighbourhood Depth. To quantify the algorithmic performance, the resulting segmentations were compared to a 'gold standard' image using three metrics viz., Sensitivity, Specificity and Accuracy. In practise the manually segmented image is also subjected to error, since even a skilled human observer will incorrectly annotate certain pixels.

\begin{tabular}{c|c|c|}
\multicolumn{1}{c}{} & \multicolumn{1}{c}{ Aneurysm Present } & Aneurysm Absent \\
\cline { 2 - 3 } Aneurysm Detected & TP & FP \\
\cline { 2 - 3 } Aneurysm Not Detected & FN & TN \\
\cline { 2 - 3 } & &
\end{tabular}

The Sensitivity and Specificity of the segmentation [30] are defined as

$$
\begin{aligned}
& \text { Sensitivity }=\frac{T P}{T P+F N} \\
& \text { Specificity }=\frac{T N}{F P+T N}
\end{aligned}
$$

A perfect detection will yield Sensitivity and Specificity values equal to unity, any real algorithm falls short of the ideal. At optimum choice, it yields Specificity=1 and Sensitivity=1.

The parameters are optimized sequentially since they appear in the algorithm in order. First parameter to be optimized is Hierarchy Height. Figure 6 shows that parameter Hough Hierarchy saturates after value 15, so it saves computation time by generating Hough Hierarchy of lesser height. Parameter that immediately follows Hough Hierarchy is Neighborhood Depth, whose optimum value comes at 1 as shown in fig. 7 . Though not implemented in this paper but intricate survey of the algorithm reveals that Neighborhood Depth should be altered for each peak number $\left(n_{p}\right)$. Next parameter to be optimized shown in fig. 8 is Peak Depth, whose optimum value comes at a value of 3 , below which more non-aneurysm detected regions will appear which in turn would increase the size of the buffer memory requirement. Optimization of the remaining two parameters $C$ and $k$ are interchangeable. Optimum value of the parameter 'Compactness Factor' $(C)$ shown in 
fig. 9 is within the range of $[0.35,0.6]$ from which we have chosen 0.55 as optimum value in our experiment. It is clear from the range of optimum values of $C$ that cerebral aneurysms are no way ideally circular, instead it shows greater tendency to be ellipsoidal. Finally the parameter $k$ plotted in fig. 10used in eq. (39) for evaluation of minimum area, optimizes at a value of 3 . These optimum values are listed in the table 2 .

The goal of this research is to accurately detect and access the type of aneurysms. Since, our visual system is biased towards radial distance of the aneurysm, we propose here a new metric for measurement of the accuracy of the detected aneurysms. Here, the manually segmented ground truth aneurysm portions $(\mathbb{O})$ are taken, whose Euclidean distance transform are computed. It is then normalized $\left(\mathbb{O}_{\xi n}\right)$ and then percentage of common pixels with the detected segmented aneurysm regions $(\mathbb{A})$ are recorded.

$$
\begin{aligned}
\mathbb{O}_{\xi} & =\text { Euclidean Distance Transform }(\mathbb{O}) \\
\mathbb{O}_{\xi n} & =\frac{\mathbb{O}_{\xi}}{\max \left(\mathbb{O}_{\xi}\right)} \\
\text { Accuracy }(\%) & =\left(\frac{\mathbb{O}_{\xi n} \wedge \mathbb{A}}{\mathbb{O}_{\xi n}}\right) \times 100, \text { here } \wedge \text { is implemented using min operation }
\end{aligned}
$$

There is no need to measure overflow percentage as thresholding safely truncates aneurysm maintaining a

\begin{tabular}{|c|c|c|c|}
\hline \multicolumn{2}{|c|}{ Algorithm } & Weight & Optimum value \\
\hline \multicolumn{2}{|c|}{ Hough Hierarchy } & Hierarchy Height & 15 \\
\hline \multirow{2}{*}{\multicolumn{2}{|c|}{ Peak Trekking }} & $\begin{array}{l}\text { Neighborhood } \\
\text { Depth }\end{array}$ & 1 \\
\hline & & Peak Depth & 3 \\
\hline \multirow{2}{*}{$\begin{array}{l}\text { Detection of } \\
\text { Aneurysm }\end{array}$} & Compactness Factor & C & 0.55 \\
\hline & Minimum Area & $k$ & 3 \\
\hline
\end{tabular}
safe boundary from the aneurysm wall without affecting the accuracy of the detection.

Table 2: Optimum values of different weights

The weights chosen for our experiment are shown in the Table 2. Relationship between peak number, area and compactness factor (CF) for each of these respective figures has been summarized in Tables (3a) to (3e).

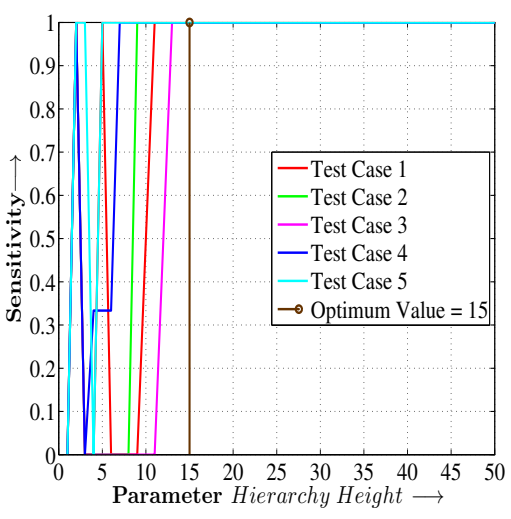

(a)

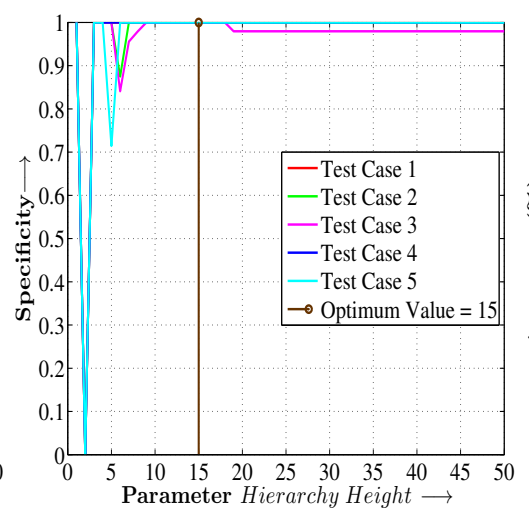

(b)

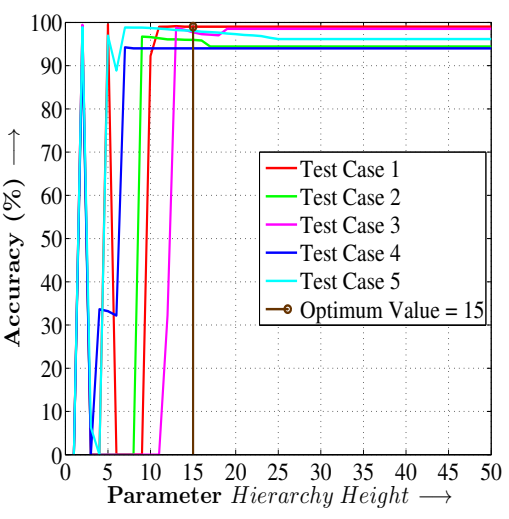

(c)

Figure 6: Optimum value selection of parameter Hierarchy Height 


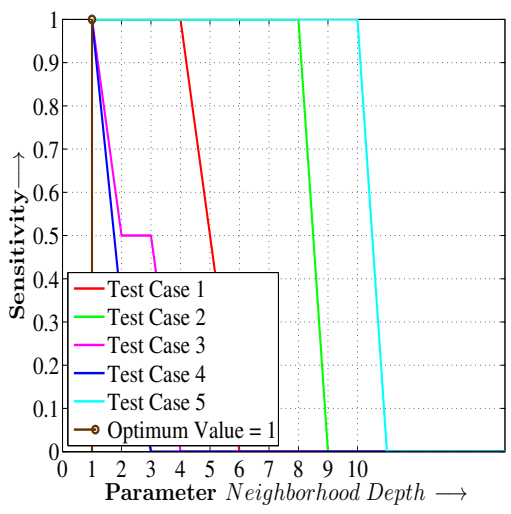

(a)

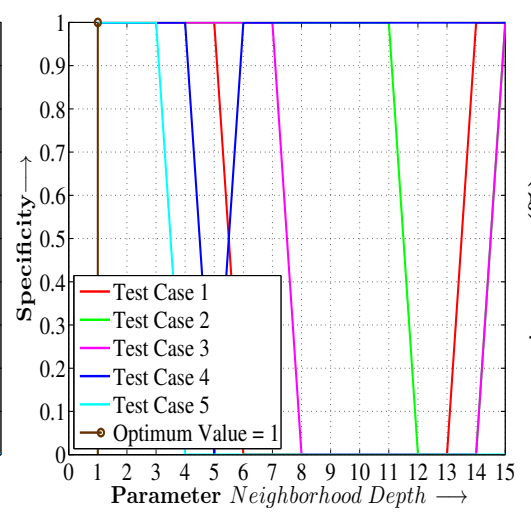

(b)

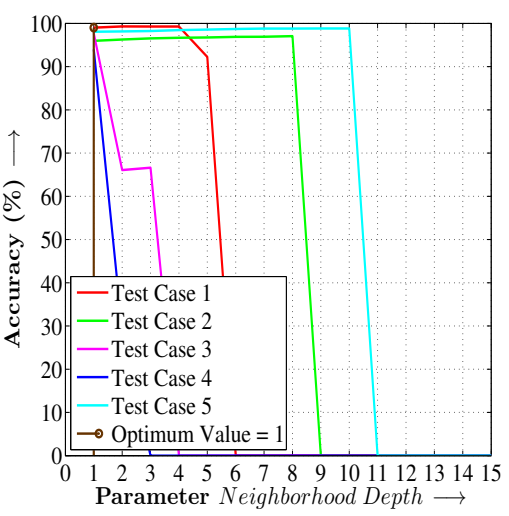

(c)

Figure 7: Optimum value selection of parameter Neighborhood Depth

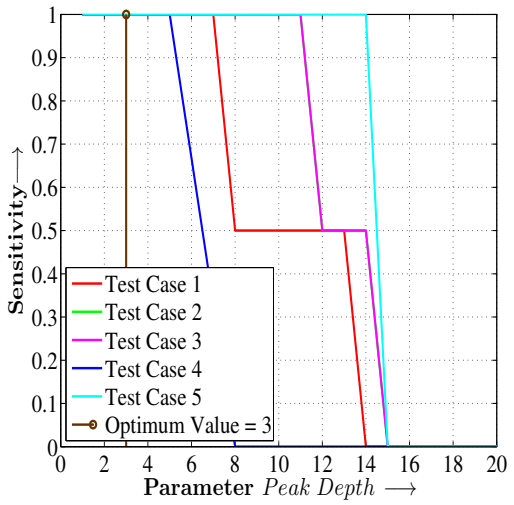

(a)

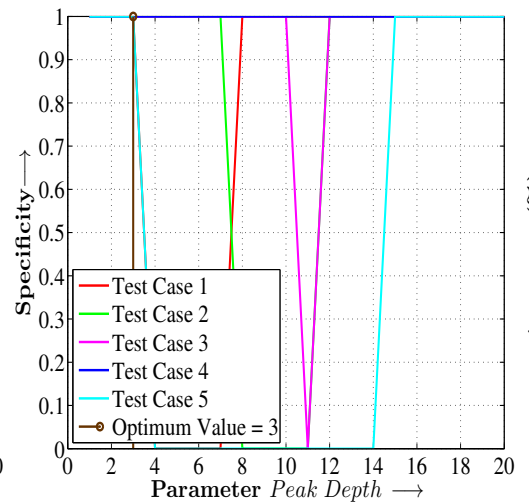

(b)

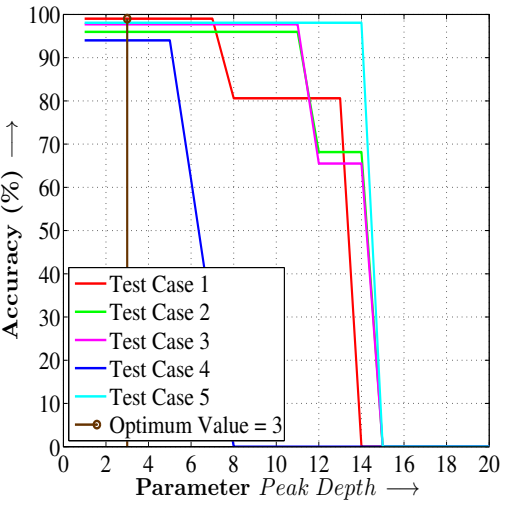

(c)

Figure 8: Optimum value selection of parameter Peak Depth

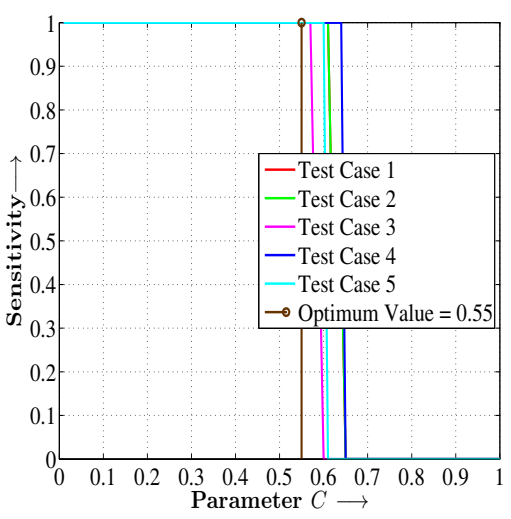

(a)

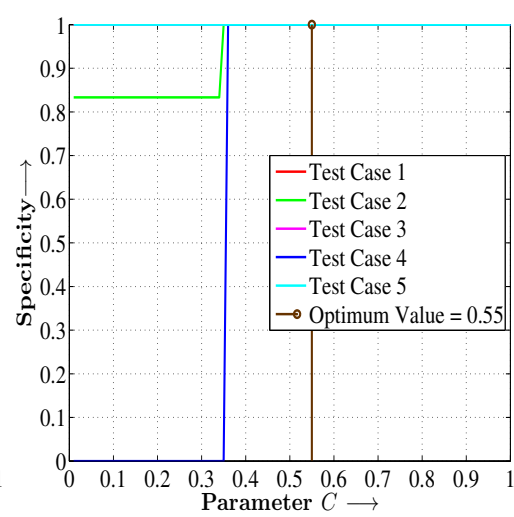

(b)

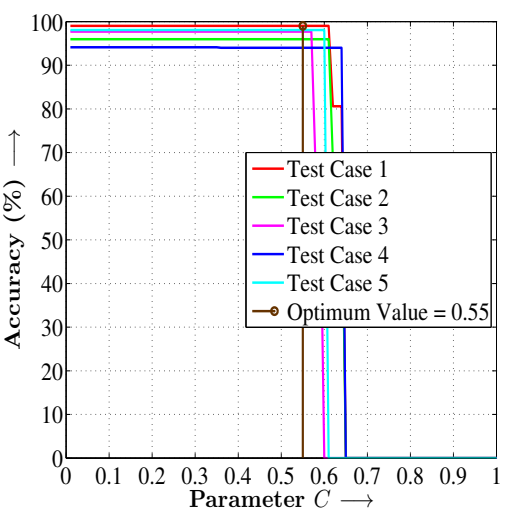

(c)

Figure 9: Optimum value selection of parameter Compactness Factor 


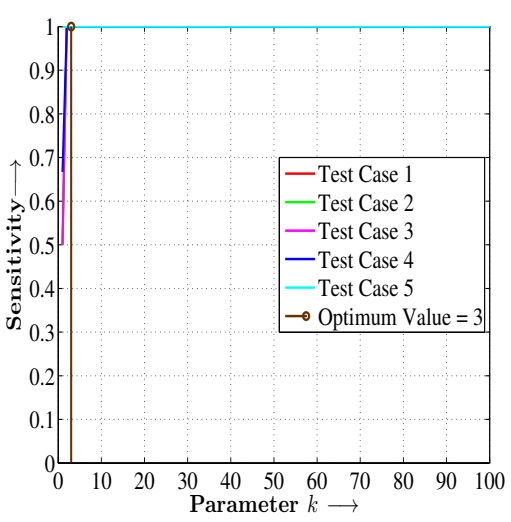

(a)

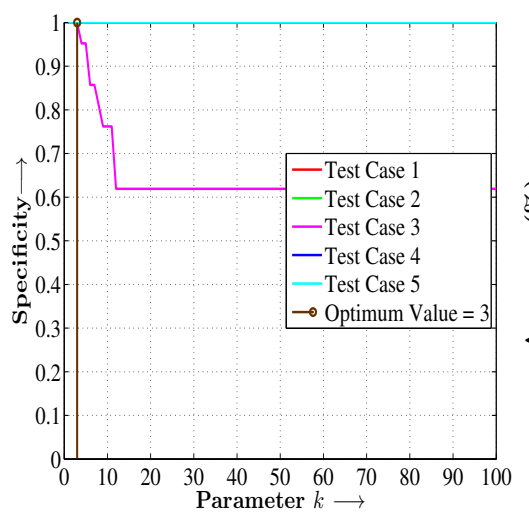

(b)

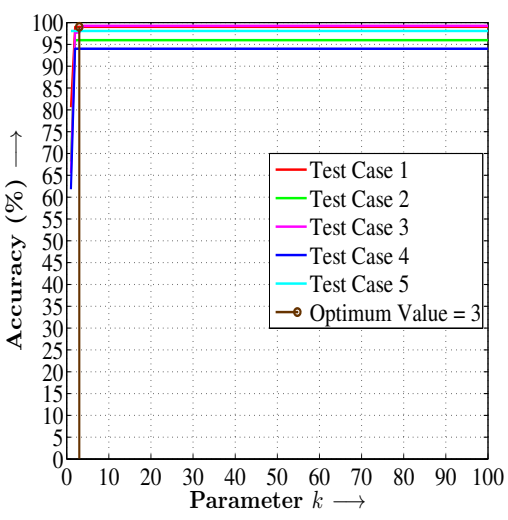

(c)

Figure 10: Optimum value selection of parameter $k$ for minimum area evaluation

\section{Simulation results}

To exhibit the competence of our proposed algorithm in detecting single and multiple cerebral aneurysms of various sizes, we have tested our algorithm over 12 different DSA test images of different types and sizes. Due to page limitation we have shown only five test images. The test images are taken from the benchmark database of Dr. Balaji Anvekar's Neuroradiology Cases [31] and Brain Aneurysms Foundations [32]. The simulation work was performed on MATLAB 7.0 software. Our achievement in properly locating the position and size of aneurysms has been shown in figures $(12)$ to $(16)$. In each of the five figures, actual DSA image $(Y)$ has been given in sub-figure (a). Grayscale image $(Y)$ segmented to binary image $(I)$ by the process of Auto-threshold is shown in sub-figure (b). The Hough Hierarchy 3D parameter space $(\mathbb{H})$ generated from Modified Hough Circle transform is shown as mesh diagram in sub-figure (e). The detected regions $(\mathbb{D})$ generated from Peak Trekking algorithm are marked chromatically with increasing peak number $\left(n_{p}\right)$ over the original image in sub-figure (c). The aneurysm regions $\mathbb{A}$ that are detected and classified from the detected regions $(\mathbb{D})$ are shown in sub-figure (d). The chromatic map used is shown in sub-figure (11a). If the chromatic map colors are exhausted while marking the regions, it is repeated with lesser luminance for the next shapes. Sometime detected regions can be overlapping, those colors are defined by chromatic overlap map as shown in sub-figure (11b). Detection and classification of aneurysms for first 5 generated shapes are shown in tables (3a) to (3e), along with parameters 'Peak No', 'Area', 'CF' and 'Diameter'. The scaling factor for each image are given in the table footnote.

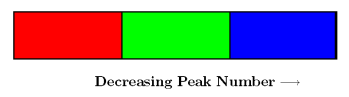

(a) Chromatic map

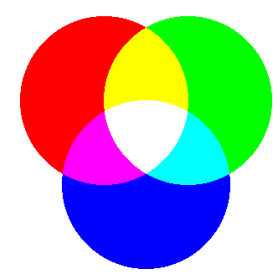

(b) Chromatic overlap map

Figure 11: Color coding used for aneurysm depiction 


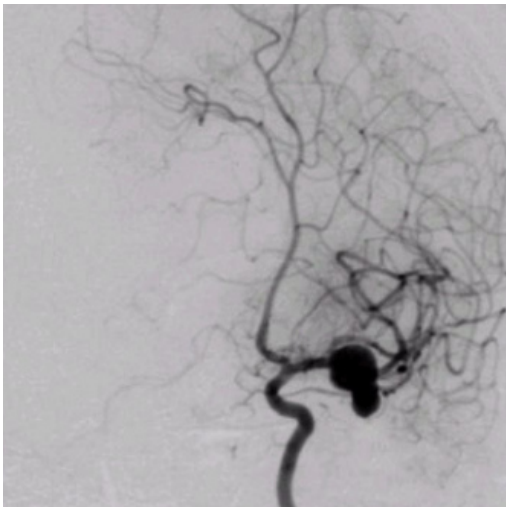

(a)

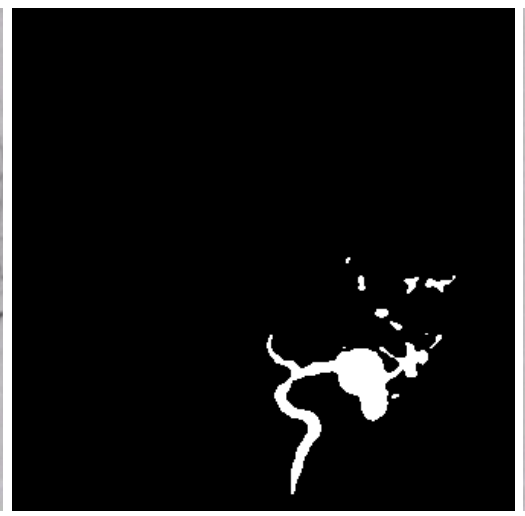

(b)

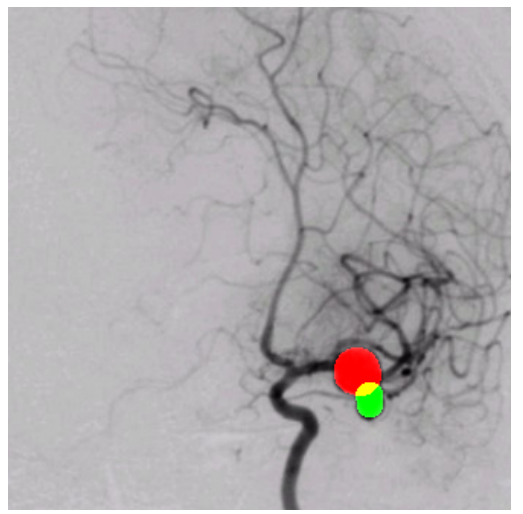

(d)

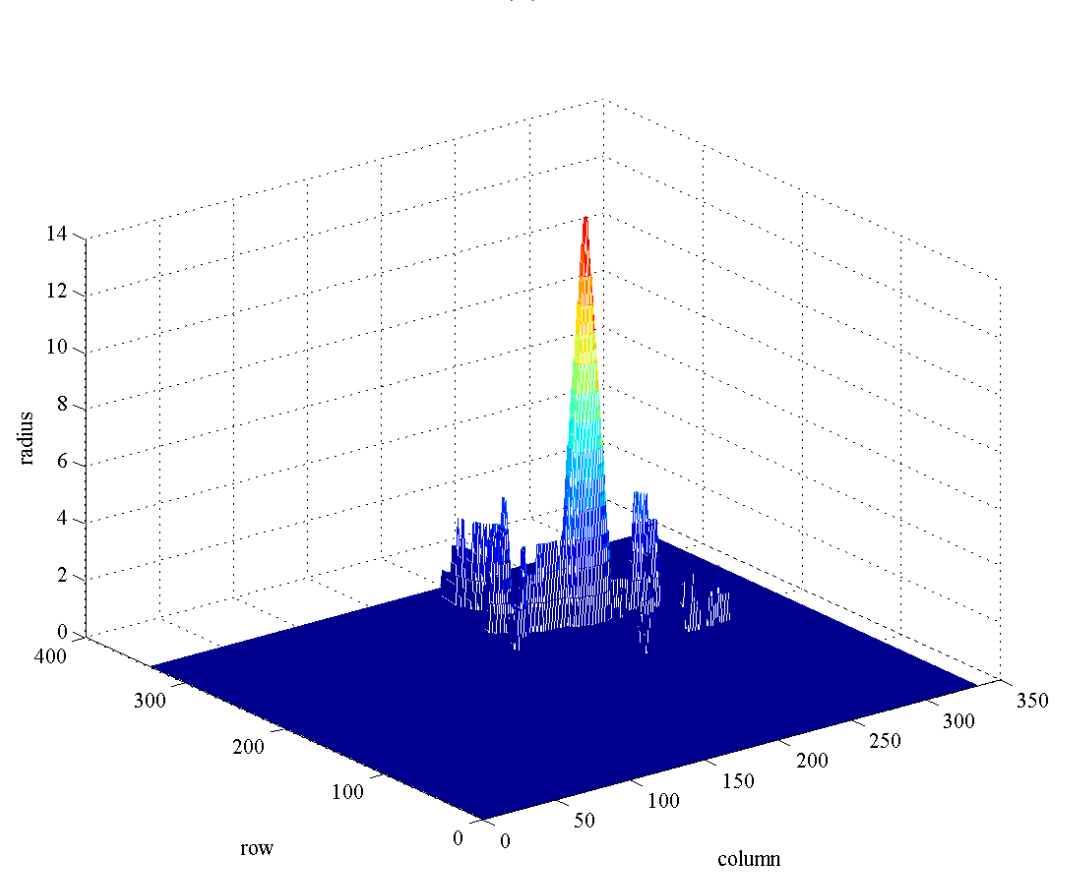

(e)

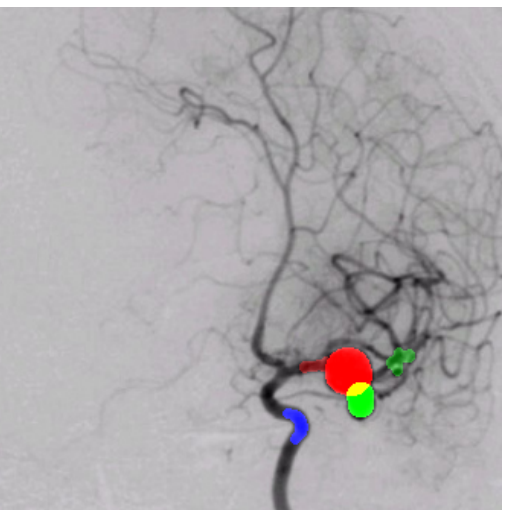

(c)

Figure 12: Detection of cerebral aneurysm for 1st test image[31] 


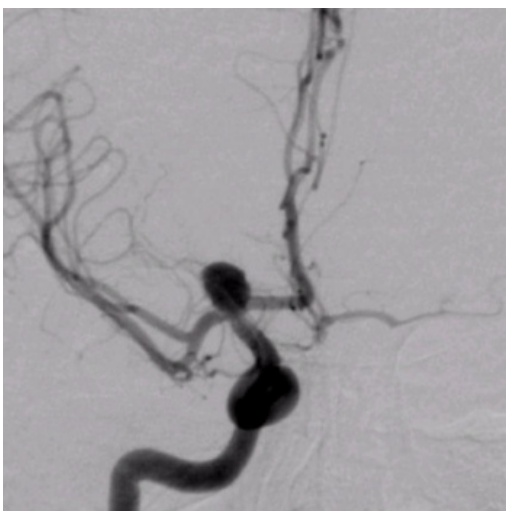

(a)

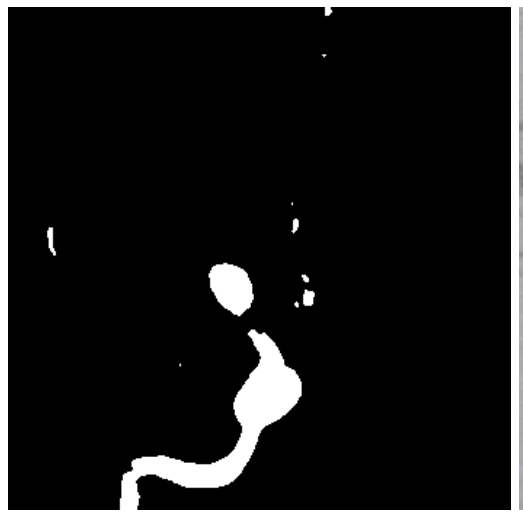

(b)

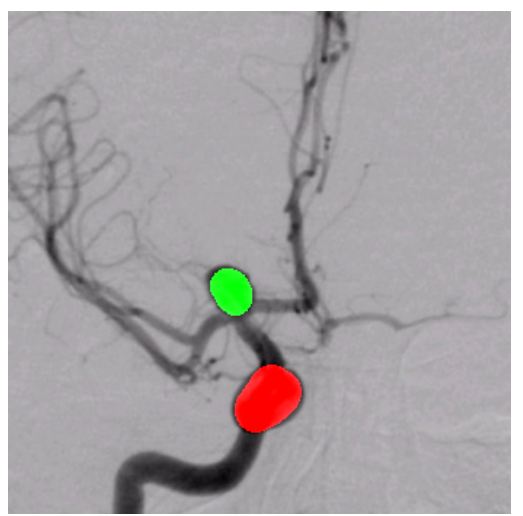

(d)

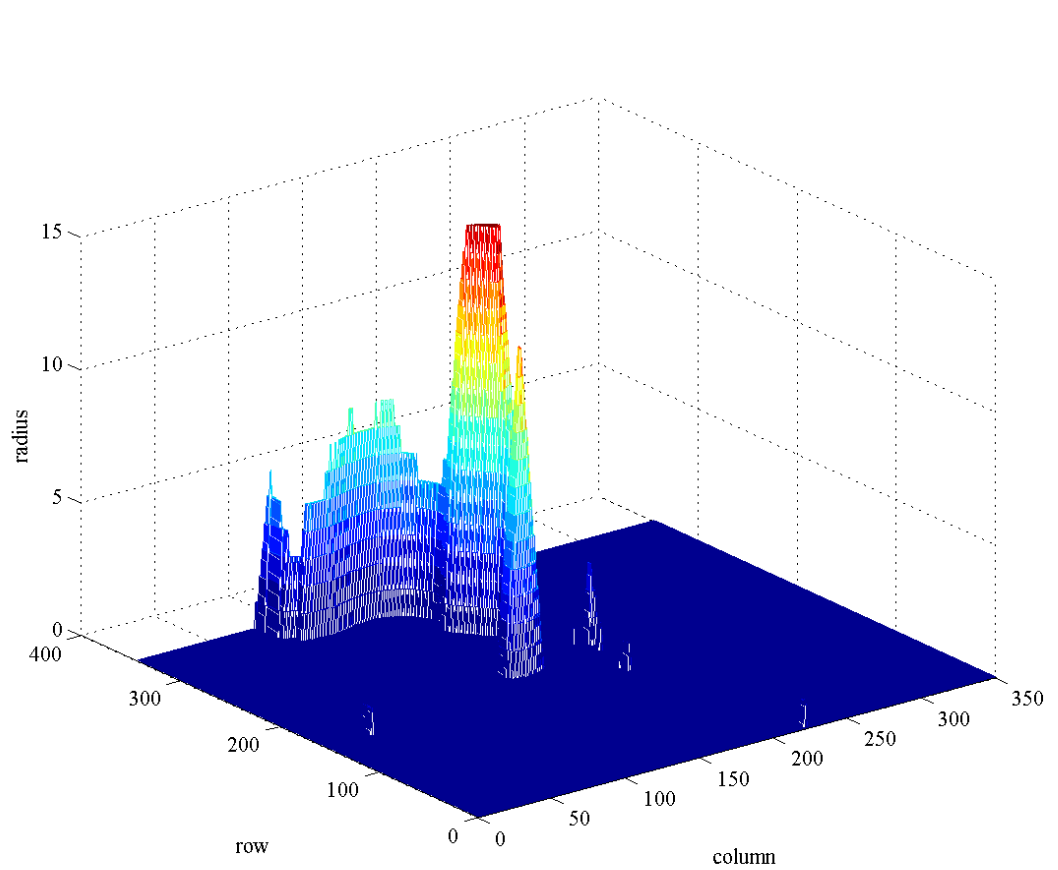

(e)

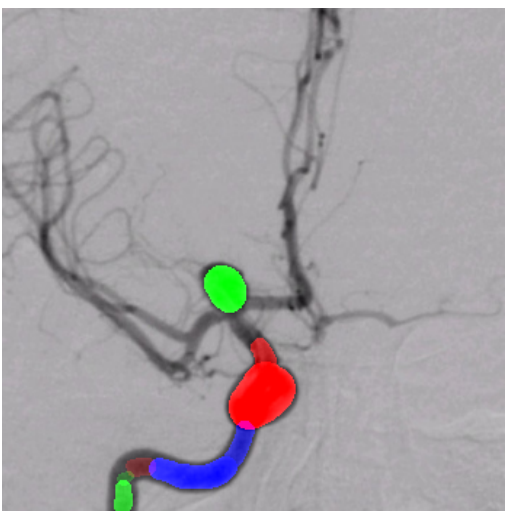

(c)

Figure 13: Detection of cerebral aneurysm for 2nd test image[31] 


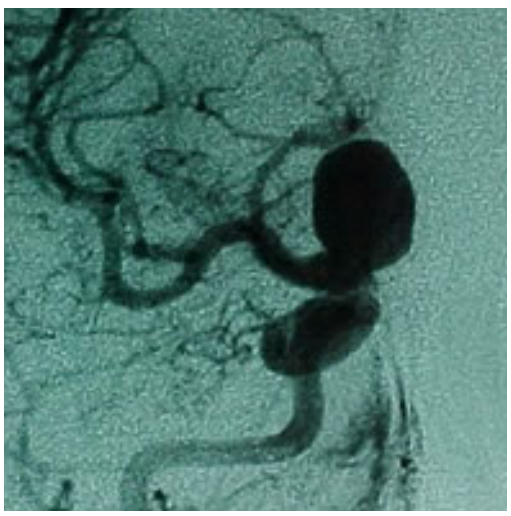

(a)

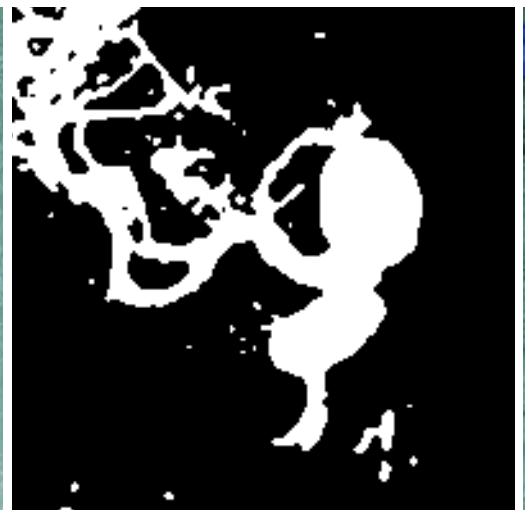

(b)

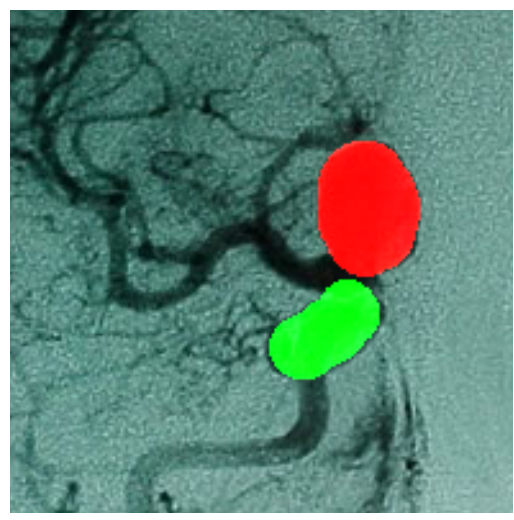

(d)

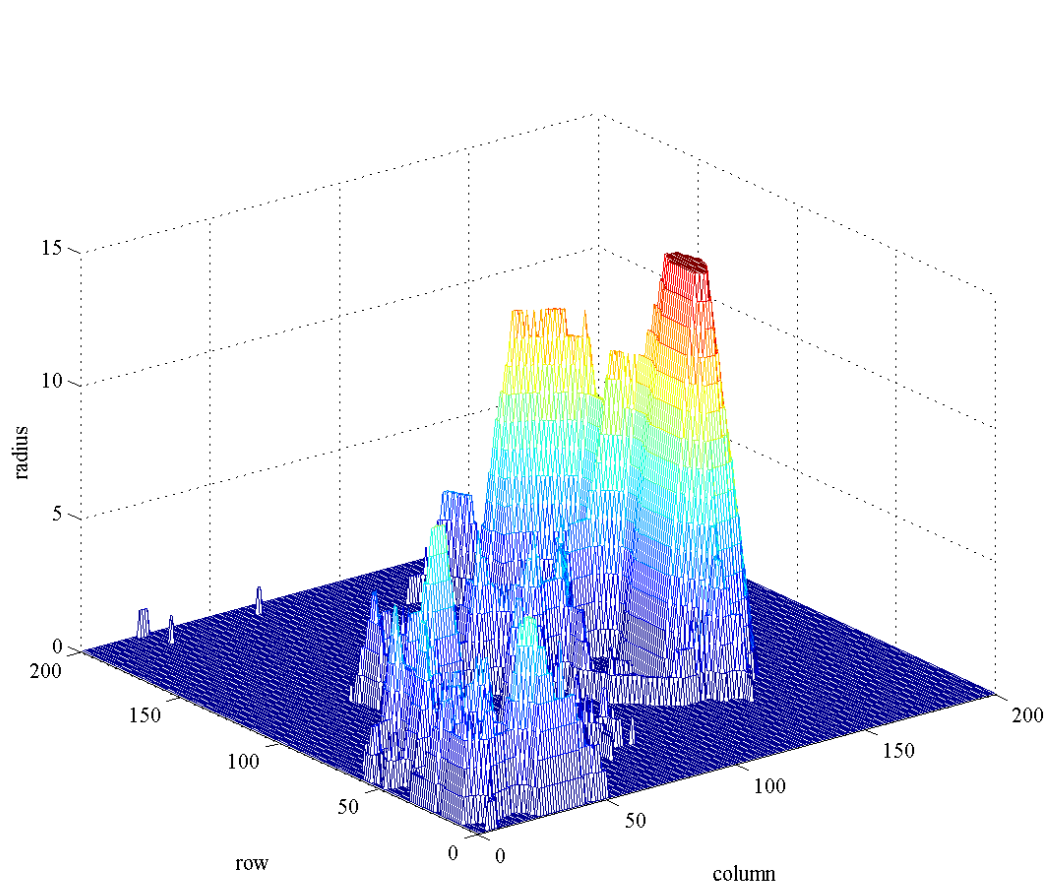

(e)

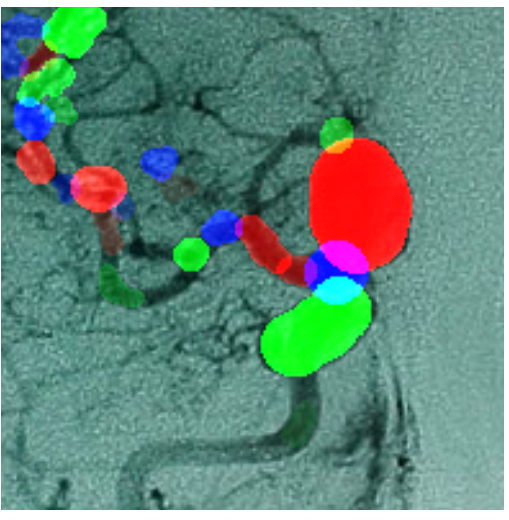

(c)

Figure 14: Detection of cerebral aneurysm for 3rd test image[32] 


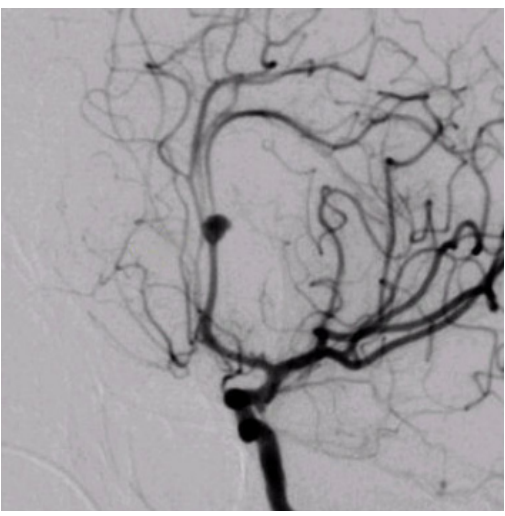

(a)

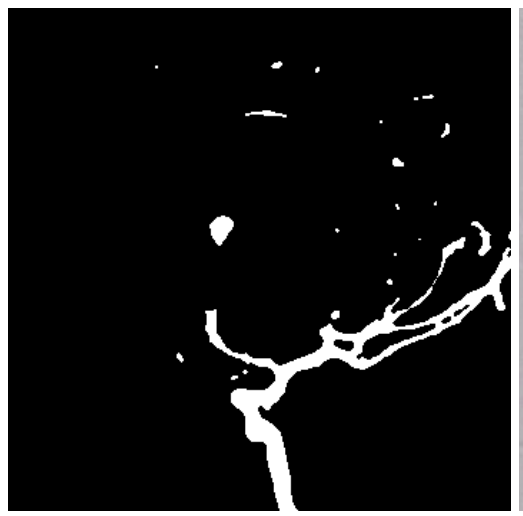

(b)

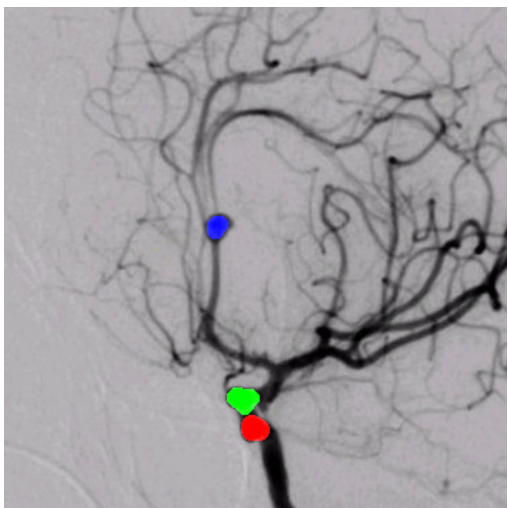

(d)

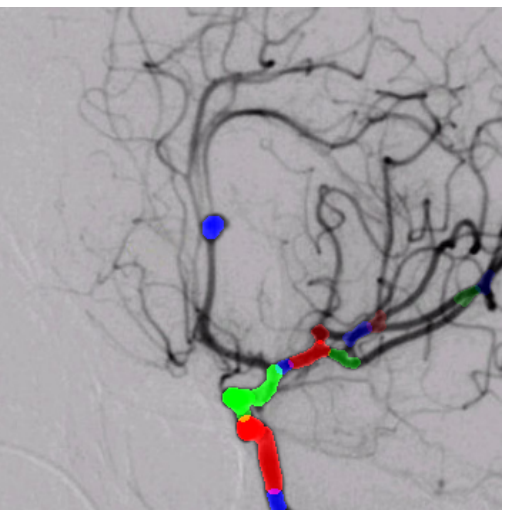

(c)

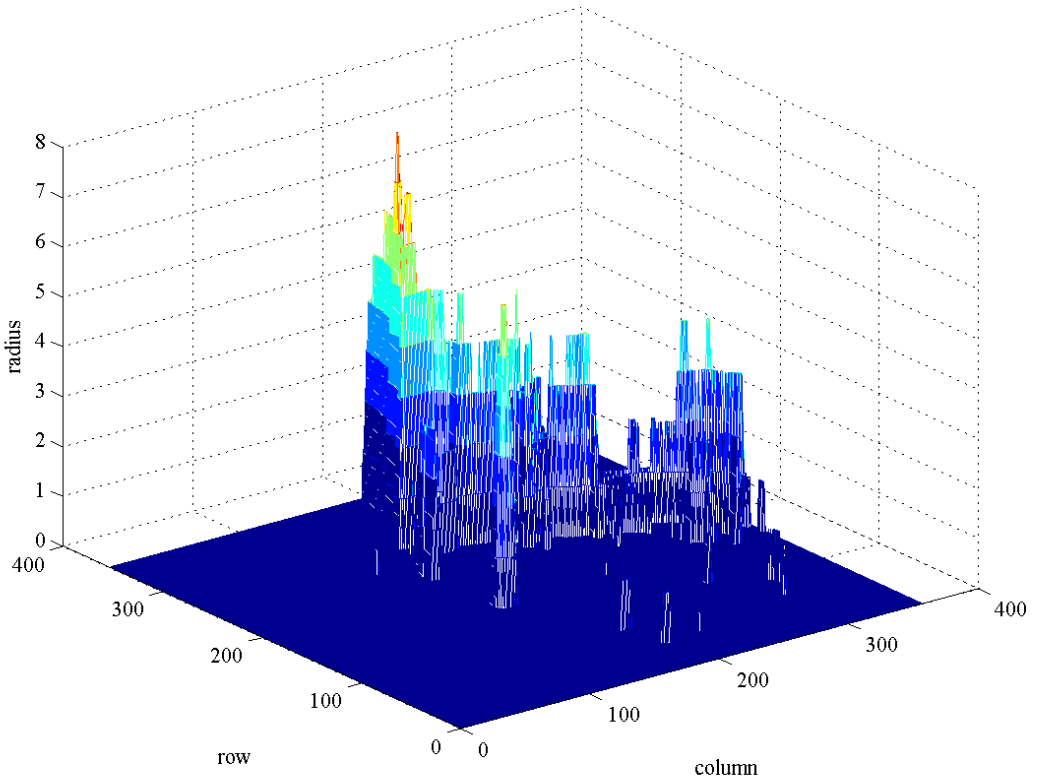

(e)

Figure 15: Detection of cerebral aneurysm for 4th test image[31] 


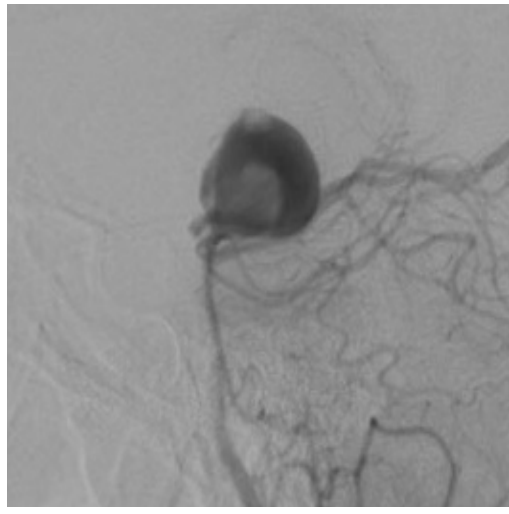

(a)

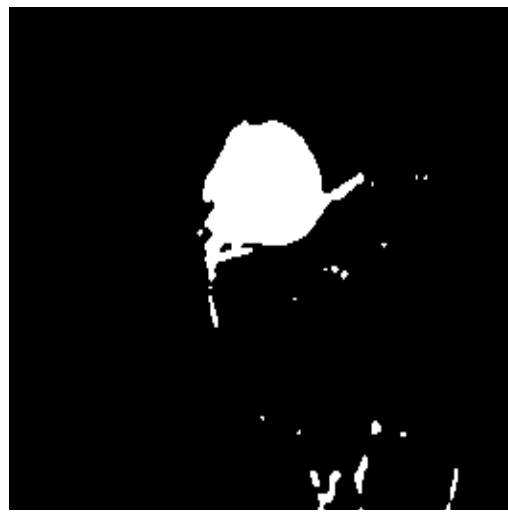

(b)

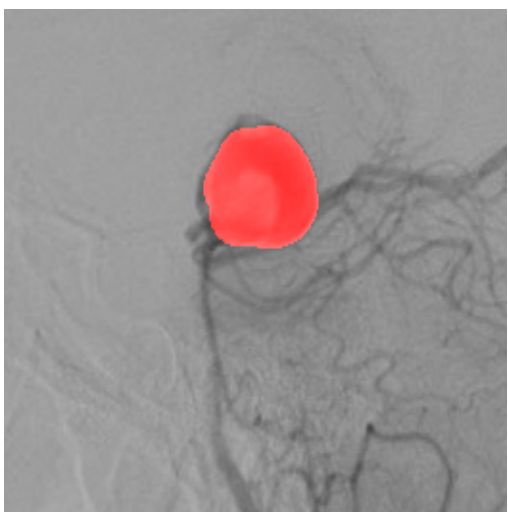

(d)

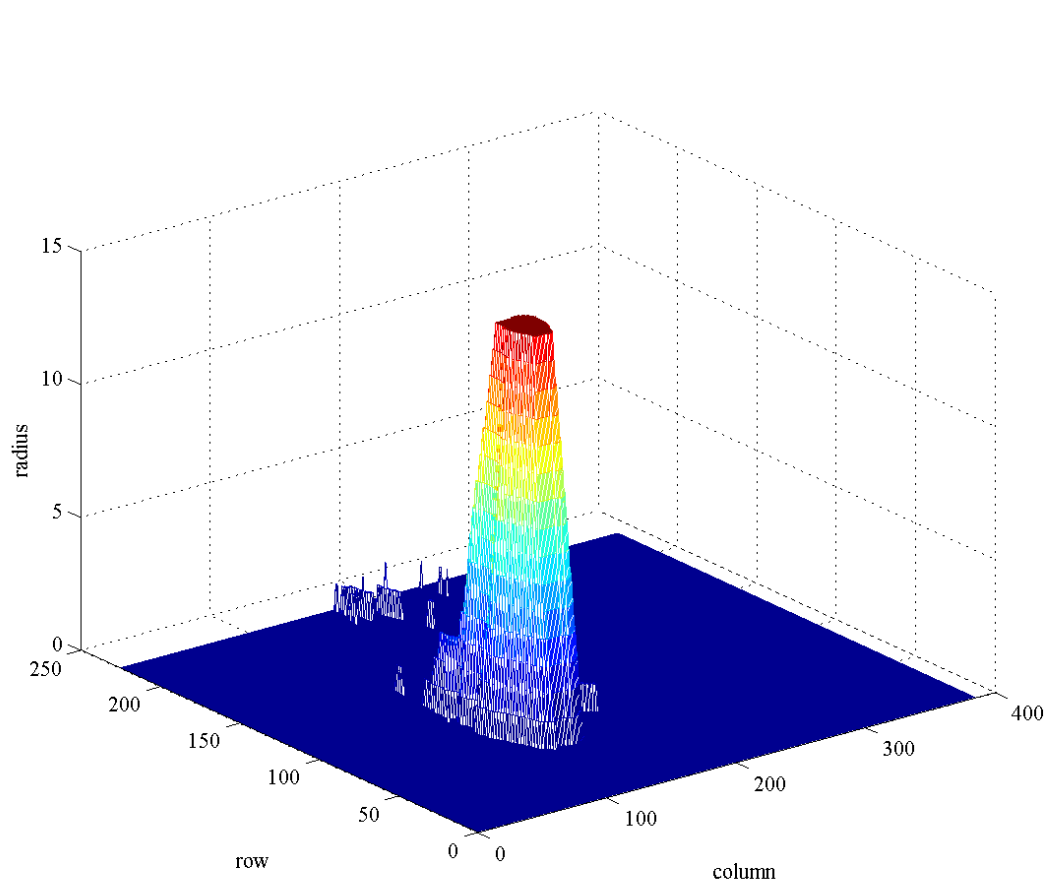

(e)

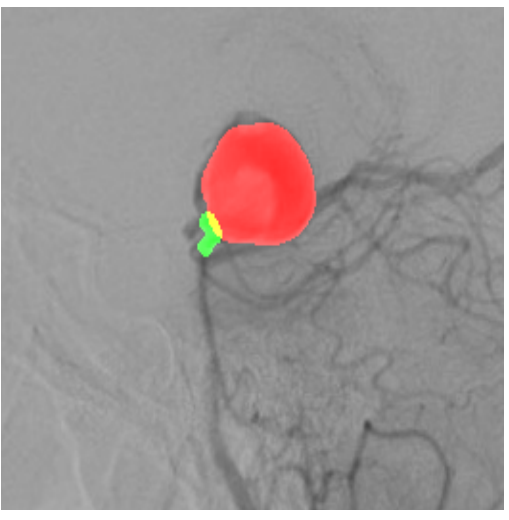

(c)

Figure 16: Detection of cerebral aneurysm for 5th test image[31] 
Table 3: Classification and Detection of aneurysms on the basis of four parameters

(a) For 1 st test image $\mathrm{a}^{a}[$ Figure 12,

\begin{tabular}{cccccc}
\hline Peak No & Area & CF & Is Aneurysm & Diameter $(\mathbf{m m})$ & Aneurysm Type \\
\hline 1 & 793 & 0.64 & True & 19.07 & Large \\
2 & 350 & 0.61 & True & 12.67 & Medium \\
3 & 222 & 0.43 & False & 10.09 & - \\
4 & 157 & 0.43 & False & 8.48 & - \\
5 & 209 & 0.47 & False & 9.79 & - \\
\hline
\end{tabular}

${ }^{a}$ Scaling Factor : 0.6

(b) For 2nd test image $]^{b}[$ Figure 13

\begin{tabular}{cccccc}
\hline Peak No & Area & CF & Is Aneurysm & Diameter $(\mathbf{m m})$ & Aneurysm Type \\
\hline 1 & 1496 & 0.64 & True & 17.46 & Large \\
2 & 711 & 0.62 & True & 12.04 & Medium \\
3 & 958 & 0.35 & False & 13.97 & - \\
4 & 287 & 0.62 & False & 7.65 & - \\
5 & 248 & 0.55 & False & 7.11 & - \\
\hline
\end{tabular}

${ }^{b}$ Scaling Factor : 0.4

(c) For 3rd test imag $\rfloor^{C}[$ Figure 14

\begin{tabular}{cccccc}
\hline Peak No & Area & CF & Is Aneurysm & Diameter $(\mathbf{m m})$ & Aneurysm Type \\
\hline 1 & 1689 & 0.60 & True & 27.82 & Giant \\
2 & 1080 & 0.57 & True & 22.25 & Large \\
3 & 485 & 0.66 & False & 14.91 & - \\
4 & 317 & 0.66 & False & 12.05 & - \\
5 & 403 & 0.66 & False & 13.59 & - \\
\hline
\end{tabular}

${ }^{c}$ Scaling Factor : 0.6

(d) For 4th test imag ${ }^{d}[$ Figure 15]

\begin{tabular}{cccccc}
\hline Peak No & Area & CF & Is Aneurysm & Diameter $(\mathbf{m m})$ & Aneurysm Type \\
\hline 1 & 289 & 0.65 & True & 5.75 & Small \\
2 & 315 & 0.68 & True & 6.01 & Small \\
3 & 194 & 0.69 & True & 4.71 & Small \\
4 & 584 & 0.36 & False & 8.18 & $\ldots$ \\
5 & 348 & 0.45 & False & 6.31 & - \\
\hline
\end{tabular}

${ }^{d}$ Scaling Factor : 0.3

(e) For 5th test image $\rfloor^{e}[$ Figure 16]

\begin{tabular}{cccccc}
\hline Peak No & Area & CF & Is Aneurysm & Diameter $(\mathbf{m m})$ & Aneurysm Type \\
\hline 1 & 2587 & 0.60 & True & 34.44 & Giant \\
2 & 168 & 0.52 & False & 8.78 & $\ldots$ \\
\hline
\end{tabular}

${ }^{e}$ Scaling Factor : 0.6 
Table 4: Performance Analysis of MHCT-PT technique

\begin{tabular}{cccc}
\hline Test Image Number & Sensitivity & Specificity & Accurate Match (\%) \\
\hline 1st & 1 & 1 & 99.0094 \\
2nd & 1 & 1 & 95.9682 \\
3rd & 1 & 1 & 97.7180 \\
4th & 1 & 1 & 93.9899 \\
5th & 1 & 1 & 98.0625 \\
\hline
\end{tabular}

As can be observed from the processed images of figures $(12)$ to $(16)$ and performance analysis in table 4 our proposed approach is capable enough in finding out the appearance, type and proper location of aneurysm. Moreover, it is very much efficient in highlighting the affected area with a higher degree of accuracy. Even for multiple CA, MHCT-PT technique can successfully identify and even classify all the aneurysms. Altogether, this has made the proposed solution very much attractive in the field of medical imaging.

\section{Comparison}

We compare our aneurysm detection methodology against three works as shown in the table 5. Inspired by the works of Parikh et al. [33], here parameters 'sensitivity', 'specificity' and 'accuracy' are employed to evaluate the efficacy of the aneurysm detection methods.

Table 5: Comparison of our MHCT-PT method with other state of the art methods

\begin{tabular}{cccccc}
\hline References & Sensitivity & Specificity & Accuracy & User Interaction & Modality \\
\hline Proposed method & 1 & 1 & 96.9 & Automatic & DSA \\
Li et al. [34] & 0.99 & 1 & 99.4 & Interactive & DSA \\
Villablanca et al. [8] & 0.99 & 1 & 99.5 & Interactive & DSA \\
McKinney et al. [6] & 0.974 & 0.9 & 95.8 & Semi-Automatic & Multisection \\
& & & & & CT Angiography \\
Hentschke et al. [35] & 0.95 & - & - & Automatic & 3D-RA \\
& 1 & - & - & & MRA \\
& 0.95 & - & - & & CTA \\
\hline
\end{tabular}

\section{Conclusion}

Cerebral aneurysm can successfully be detected by our proposed approach of Peak Trekking of Hough Hierarchy mountain generated from Modified Hough Circle Transform over Auto-Thresholded DSA grayscale image. However, proper selection of user-defined parameters is very much crucial and therefore needs manual tuning. Optimum selection of these values can lead to accurate detection of any type of aneurysm \& even abnormal vessels. Future research may be carried out in the direction of detecting the neck of the aneurysms and also optimizing the weights with a higher degree of precision by varying those parameters in an adaptive way like by means of neural network. 


\section{References}

[1] G. Roberto and L. Roberto, "Intracranial aneurysms," Essentials of Neuroradiology - CC ECR 2005, vol. 15, pp. 441-447, 2005. DOI: $10.1007 / \mathrm{s} 00330-004-2614-8$ (cit. on p. 57)

[2] E. F. Wijdicks, D. F. Kallmes, E. M. Manno, J. R. Fulgham, and D. G. Piepgras, "Subarachnoid hemorrhage: neurointensive care and aneurysm repair," Mayo Clinic Proceedings, vol. 80, pp. 550-559, 2005, PubMed: 15819296 (cit. on p. 57)

[3] C. M. Strother, F. Bender, Y. Deuerling-Zheng, K. Royalty, K. A. Pulfer, J. Baumgart, M. Zellerhoff, B. Aagaard-Kienitz, D. B. Niemann, and M. L. Lindstrom, "Parametric Color Coding for Digital Subtraction Angiography," American Journal of Neuroradiology, vol. 31, pp. 919-924, 2010. DOI: $10.3174 /$ ajnr.A2020 (cit. on p. 57)

[4] C. M. Hentschke, O. Beuing, R. Nickl, and K. Tonnies, "Automatic cerebral aneurysm detection in multimodal angiographic images," in Nuclear Science Symposium and Medical Imaging Conference (NSS/MIC), IEEE, 2011, pp. 3116-3120 (cit. on p. 58)

[5] C. M. Hentschke, O. Beuing, R. Nickl, and K. D. Tönnies, "Detection of cerebral aneurysms in MRA, CTA and 3D-RA data sets," in SPIE Medical Imaging, International Society for Optics and Photonics, 2012, pp. 83151I-83151I (cit. on p. 58)

[6] A. McKinney, C. Palmer, C. Truwit, A Karagulle, and M Teksam, "Detection of aneurysms by 64-section multidetector CT angiography in patients acutely suspected of having an intracranial aneurysm and comparison with digital subtraction and 3D rotational angiography," American Journal of Neuroradiology, vol. 29, no. 3, pp. 594-602, 2008 (cit. on pp. 58, 82)

[7] L. Lu, L. J. Zhang, C. S. Poon, S. Y. Wu, C. S. Zhou, S. Luo, M. Wang, and G. M. Lu, "Digital subtraction CT angiography for detection of intracranial aneurysms: comparison with three-dimensional digital subtraction angiography," Journal of Radiology, vol. 262, pp. 605-612, 2012, PubMed:22143927 (cit. on pp. 58,59

[8] J. P. Villablanca, R. Jahan, P. Hooshi, S. Lim, G. Duckwiler, A. Patel, J. Sayre, N. Martin, J. Frazee, J. Bentson, et al., "Detection and characterization of very small cerebral aneurysms by using 2D and 3D helical CT angiography," American journal of neuroradiology, vol. 23, no. 7, pp. 1187-1198, 2002 (cit. on pp. 58, 82)

[9] J. Russell and R. Cohn, Cerebral aneurysm. Book on Demand Ltd, 2012 (cit. on p. 58)

[10] Manual on Contrast Media, 7th ed., American College of Radiology, 2010 (cit. on p. 59)

[11] D. L. Longo, A. S. Fauci, D. L. Kasper, S. L. Hauser, J. L. Jameson, and J. Loscalzo, Harrison's Principles of Internal Medicine, 18th. McGraw-Hill Education (Asia), 2012 (cit. on p. 59]

[12] G. B. Bradac, Cerebral Angiography: Normal Anatomy and Vascular Pathology, 1st. Springer, 2011, ISBN: 3642156770 (cit. on p. 59)

[13] M. Sezgin and B. Sankur, "Survey over Image Thresholding Techniques and Quantitative Performance Evaluation," Journal of Electronic Imaging, vol. 13, pp. 146-165, 2004 (cit. on p. 59)

[14] J. Mitra and A. Chandra, "Detection of Cerebral Aneurysm by Performing Thresholding-Spatial FilteringThresholding Operations on Digital Subtraction Angiogram," in Second International Conference on Digital Image Processing and Pattern Recognition. (DPPR-2012), Chennai: Springer, 2012 (cit. on p.59)

[15] P. Mildenberger, M. Eichelberg, and E. Martin, "Introduction to the DICOM standard," European Radiology, vol. 12, no. 4, pp. 920-927, 2002 (cit. on p. 59)

[16] A. F. Frangi, W. J. Niessen, K. L. Vincken, and M. A. Viergever, "Multiscale vessel enhancement filtering," in Medical Image Computing and Computer-Assisted Interventation-MICCAI 98, Springer, 1998, pp. 130-137 (cit. on p. 60) 
[17] Q. Li, S. Sone, and K. Doi, "Selective enhancement filters for nodules, vessels, and airway walls in two-and three-dimensional CT scans," Medical physics, vol. 30, p. 2040, 2003 (cit. on p. 60)

[18] N. Otsu, "A threshold selection method from gray-level histograms," Automatica, vol. 11, no. 285-296, pp. 23-27, 1975 (cit. on pp. 61, 63)

[19] J. Sauvola and M. Pietikäinen, "Adaptive document image binarization," Pattern Recognition, vol. 33, no. 2, pp. 225-236, 2000 (cit. on pp. 61, 63)

[20] P. V. C. Hough, "Method and means for recognizing complex patterns," US Patent, 1962 (cit. on p. 65

[21] M. Nixon and A. S. Aguado, Feature Extraction \& Image Processing for Computer Vision, 2nd. Elsevier, 2008, ISBN: 0123725380 (cit. on p. 65)

[22] C. D. Ruberto, "Generalized hough transform for shape matching," International Journal of Computer Applications, vol. 48, no. 1, 2012 (cit. on p. 65)

[23] O. Ecabert, J. Peters, H. Schramm, C. Lorenz, J. von Berg, M. J. Walker, M. Vembar, M. E. Olszewski, K. Subramanyan, G. Lavi, et al., "Automatic model-based segmentation of the heart in CT images," IEEE Transactions on Medical Imaging, vol. 27, no. 9, pp. 1189-1201, 2008 (cit. on p. 65)

[24] X. Chen, L. Lu, and Y. Gao, "A new concentric circle detection method based on Hough transform," in 7th International Conference on Computer Science \& Education (ICCSE), Melbourne, VIC, 2012, pp. 753-758 (cit. on p. 65)

[25] L. da Fona Costa and R. M. C. Jr., "Shape Classification and Analysis: Theory and Practice," in, 2nd. CRC Press, 2009, ch. 5, ISBN: 0849379296 (cit. on p. 65)

[26] D. D. Hearn, M. P. Baker, and W. Carithers, Computer Graphics with Open GL, 4th. Prentice Hall, 2010, ISBN: 0136053580 (cit. on p. 65)

[27] J. E. Bresenham, D. G. Grice, and S. C. Pi, Bi-directional display of circular arcs, US Patent 4,371,933, Feb. 1983 (cit. on p. 65)

[28] I. L. Dryden and K. V. Mardia, Statistical Shape Analysis, 1st. New York: Wiley, 1998, ISBN: 0471958166 (cit. on p.70)

[29] R. Dashti, J. Hemesniemi, M. Niemelä, J. Rinne, M. Porras, M. Lehecka, H. Shen, B. S. Albayrak, H. Lehto, P. Koroknay-Pál, et al., "Microneurosurgical management of middle cerebral artery bifurcation aneurysms," Surgical neurology, vol. 67, no. 5, pp. 441-456, 2007 (cit. on p. 71)

[30] T. Loong, "Understanding sensitivity and specificity with the right side of the brain," BMJ: British Medical Journal, vol. 327, no. 7417, p. 716, 2003 (cit. on p. 72)

[31] Dr. Balaji Anvekar's Neuroradiology Cases, Neuroradiology cases, http : / / www - yousaytoo . com/aneurysm-dsa/1896957 (cit. on pp.75,77,79,80)

[32] Brain Aneurysms Foundations, Cerebral Aneurysm resources, http : / / bafoundations . com / ImageGallery.html, 2013 (cit. on pp. 75, 78)

[33] R. Parikh, A. Mathai, S. Parikh, G. C. Sekhar, and R. Thomas, "Understanding and using sensitivity, specificity and predictive values," Indian journal of ophthalmology, vol. 56, no. 1, p. 45, 2008 (cit. on p. 82

[34] M. Li, Y. Cheng, Y. Li, C. Fang, S. Chen, W. Wang, D. Hu, and H. Xu, "Large-cohort comparison between three-dimensional time-of-flight magnetic resonance and rotational digital subtraction angiographies in intracranial aneurysm detection," Stroke, vol. 40, no. 9, pp. 3127-3129, 2009 (cit. on p. 82)

[35] C. M. Hentschke, K. Tonnies, O. Beuing, and R. Nickl, "A new feature for automatic aneurysm detection," in 9th IEEE International Symposium on Biomedical Imaging (ISBI), IEEE, 2012, pp. 800-803 (cit. on p. 82) 